\title{
The Spectral Web of stationary plasma equilibria: I. General theory
}

\author{
J.P. Goedbloed \\ DIFFER - Dutch Institute for Fundamental Energy Research, \\ De Zaale 20, 5612 AJ Eindhoven, the Netherlands
}

(Dated: December 17, 2017)

\begin{abstract}
A new approach to computing the complex spectrum of magnetohydrodynamic (MHD) waves and instabilities of moving plasmas is presented. It is based on the concept of the Spectral Web, exploiting the self-adjointness of the generalized Frieman-Rotenberg force operator, $\mathbf{G}$, and the Doppler-Coriolis gradient operator parallel to the velocity, $U$. The problem is solved with an open boundary, where the complementary energy $W_{\text {com }}$ represents the amount of energy to be delivered to or extracted from the system to maintain harmonic time-dependence. The eigenvalues are connected by a system of curves in the complex $\omega$-plane, the solution path and the conjugate path (where $W_{\text {com }}$ is real or imaginary) which together constitute the Spectral Web, having a characteristic geometry that has to be clarified yet, but that has a deep physical significance. It is obtained by straightforward contour plotting of the two paths. The complex eigenvalues, within a specified rectangle of the complex $\omega$-plane, are found by fast, reliable and accurate iterations. Real and Complex Oscillation Theorems, replacing the familiar tool of counting nodes of eigenfunctions, provide an associated mechanism of mode tracking along the two paths. The Spectral Web method is generalized to toroidal systems and extended to include a resistive wall by accounting for the dissipation in such a wall. It is applied in an accompanying Paper II to a multitude of the basic fundamental instabilities operating in cylindrical plasmas.
\end{abstract}

PACS numbers: 52.30.Cv, 52.30.-q, 52.35.Bj, 47.65.-d 


\section{INTRODUCTION}

The development of MHD stability theory of laboratory and astrophysical plasmas with sizeable background flows, initiated long ago, has severely suffered from the wide spread misunderstanding that this theory necessarily involves non-self-adjoint operators. The new theory of the Spectral Web presented here, on the contrary, is entirely constructed on the basis of the two quadratic forms of the potential energy and the averaged Doppler-Coriolis shift, both involving a self-adjoint operator. This approach finally provides order in the bewildering variety of complex eigenvalues that are obtained from high resolution spectral codes. This order obtains from a monotonicity property of the eigenvalues in the complex plane along the two sets of curves that constitute the Spectral Web. Such a property was long thought to be restricted to the real eigenfunctions of static equilibria, but it has now been generalized for the complex eigenfunctions of stationary equilibria. The monotonicity can not be based on node counting of the eigenfunctions, but it involves a quantity called the complementary energy which represents the energy needed to sustain the Doppler-Coriolis shifted frequencies of the instabilities. Thus, the full complex spectrum of stationary plasmas is obtained together with a connecting structure. This permits to consider the enormous diversity of MHD instabilities of laboratory and astrophysical plasmas with arbitrary flow and rotation profiles from a single unifying viewpoint. In this paper and the companion paper, ${ }^{1}$ to be referred to as Paper II, the method is introduced and illustrated with numerous instabilities of equilibria subjected to shear flow and rotation.

\section{THE SPECTRAL WEB METHOD}

\section{A. Basic spectral theory of stationary equilibria}

To highlight the new approach to the spectrum of waves and instabilities of stationary equilibria, we first summarize the basic facts of the standard formalisms for static and stationary equilibria. The common starting point is the set of ideal MHD equations, expressing conservation of mass, momentum, entropy, and magnetic flux, respectively:

$$
\begin{aligned}
& \frac{\partial \rho}{\partial t}+\nabla \cdot(\rho \mathbf{v})=0, \\
& \rho\left(\frac{\partial \mathbf{v}}{\partial t}+\mathbf{v} \cdot \nabla \mathbf{v}\right)+\nabla p+\rho \nabla \Phi_{\mathrm{gr}}-(\nabla \times \mathbf{B}) \times \mathbf{B}=0 \\
& \frac{\partial p}{\partial t}+\mathbf{v} \cdot \nabla p+\gamma p \nabla \cdot \mathbf{v}=0, \\
& \frac{\partial \mathbf{B}}{\partial t}-\nabla \times(\mathbf{v} \times \mathbf{B})=0, \quad \nabla \cdot \mathbf{B}=0 .
\end{aligned}
$$

Here, $\rho$ is the density, $\mathbf{v}$ is the velocity, $p$ is the pressure, $\mathbf{B}$ is the magnetic field, and $\Phi_{\text {gr }}$ indicates the gravitational field of an external compact object (included for astrophysical applications, of course absent in applications to laboratory plasmas). All quantities are assumed to have been made dimensionless by means of some length scale $\ell_{0}$, some density measure $\rho_{0}$, and some magnetic field strength $B_{0}$, so that the vacuum permeability $\mu_{0}$ no longer appears. In this way, the equations also have become scale independent, ${ }^{2,3}$ so that they may be used to describe the dynamics of plasmas in the laboratory as well as on the huge 
scales of astrophysics. Of course, the overall spatial configurations and associated boundary conditions will be quite different. For example, confinement of plasmas in tokamaks is eventually due to magnetic fields produced by external coils that are firmly attached to the laboratory, whereas plasmas in galaxies are primarily confined by the balance of gravitational and centrifugal acceleration.

Spectral theory of MHD waves and instabilities requires a split of the equations (1)-(4) into a time-independent equilibrium part and perturbations that depend on time. Intuition on this subject has been largely based on the enormous simplification that results from the assumption of a static equilibrium $[\partial / \partial t=0$ and $\mathbf{v}=0$, so that only the last three terms of Eq. (2) remain] with perturbations proportional to $\exp (-\mathrm{i} \omega t)$. This led to a spectral problem described by the linear force operator $\mathbf{F}$ acting on the plasma displacement field $\boldsymbol{\xi}$ :

$$
\mathbf{F}(\boldsymbol{\xi})=\rho \frac{\partial^{2} \boldsymbol{\xi}}{\partial t^{2}}=-\rho \omega^{2} \boldsymbol{\xi}
$$

In particular, the associated quadratic form of the potential energy,

$$
W \equiv-\frac{1}{2} \int \boldsymbol{\xi}^{*} \cdot \mathbf{F}(\boldsymbol{\xi}) d V,
$$

gave rise to the powerful energy principle ${ }^{4,5}$ permitting a preliminary stability analysis by means of trial functions. In this way. a first 'intuitive' understanding was obtained of the different stabilizing and destabilizing effects operating in the various instabilities, like kinks, interchanges, ballooning modes, etc.

Simplicity does not necessarily lead to understanding of complex phenomena. In particular, the assumption of a static equilibrium simply makes no sense for the vast majority of astrophysical plasmas: they all move, frequently at super-Alfvénic and even relativistic speeds. Laboratory fusion plasmas usually also exhibit flows, be it at much more modest, but certainly not negligible, speeds. Hence, it would appear logical that the original static analyses would have been superseded by analyses of stationary equilibria, where $\partial / \partial t=0$ again, but $\mathbf{v} \neq 0$ so that all other terms of the four equations (1)-(4) contribute.

In fact, the appropriate spectral counterpart of Eq. (5) for stationary equilibria was developed by Frieman and Rotenberg, ${ }^{6}$ not long after the original papers on the energy principle appeared:

$$
\mathbf{G}(\boldsymbol{\xi})-2 \rho \mathbf{v} \cdot \nabla \frac{\partial \boldsymbol{\xi}}{\partial t}-\rho \frac{\partial^{2} \boldsymbol{\xi}}{\partial t^{2}}=\mathbf{G}(\boldsymbol{\xi})-2 \rho \omega U \boldsymbol{\xi}+\rho \omega^{2} \boldsymbol{\xi}=0 .
$$

Now two operators appear, viz. the generalized force operator $\mathbf{G}$ (in our notation, replacing the original symbol $\mathbf{F}$ of Ref. 6 ) and the projected gradient operator $U$ parallel to the velocity (multiplied with the obvious factor $-\mathrm{i}$, see Ref. 7):

$$
\mathbf{G}(\boldsymbol{\xi}) \equiv \mathbf{F}(\boldsymbol{\xi})+\nabla \cdot(\boldsymbol{\xi} \rho \mathbf{v} \cdot \nabla \mathbf{v})-\rho(\mathbf{v} \cdot \nabla)^{2} \boldsymbol{\xi}, \quad U \equiv-\mathrm{i} \mathbf{v} \cdot \nabla
$$

For many applications, it is appropriate to consider the system to be closed (by genuine or by fictitious walls), giving rise to the following boundary conditions (BCs) on the component $\xi \equiv \mathbf{n} \cdot \boldsymbol{\xi}$ normal to the magnetic surfaces:

$$
\begin{array}{ll}
\xi\left(x=x_{\ell}\right)=0 & \text { (inner wall, 'left' BC), } \\
\xi\left(x=x_{\mathrm{r}}\right)=0 & \text { (outer wall, 'right' BC). }
\end{array}
$$


Here, and below, the coordinate $x$ is used to label the separate flux surfaces, whereas the two other coordinates, labeling the different points on the flux surfaces, will be suppressed. The operators $\mathbf{G}$ and $U$ are both self-adjoint, provided these BCs are satisfied. Actually, the latter restriction is only needed with respect to the operator $\mathbf{G}$. The operator $U$ is always self-adjoint, irrespective of satisfaction of BCs, which is why we have called that behavior super self-adjoint. ${ }^{3}$ Due to this self-adjointness, the quadratic forms associated with $\mathbf{G}$ and $U$,

$$
W \equiv-\frac{1}{2} \int \boldsymbol{\xi}^{*} \cdot \mathbf{G}(\boldsymbol{\xi}) d V, \quad V \equiv \frac{1}{2} \int \rho \boldsymbol{\xi}^{*} \cdot U \boldsymbol{\xi} d V
$$

are both real. They represent the potential energy and the average Doppler-Coriolis shift, respectively, of the solutions of the spectral problem (7)-(10). Incidentally, the far reaching implications of the Frieman-Rotenberg spectral equation for the nonlinear plasma dynamics have been pointed out recently by Keppens and DeMaerel. ${ }^{8}$

Concerning the BCs (9) and (10): note that the non-bold symbol $\xi$ is used to single out the normal component, and that the inner wall BC (9) is to be understood as indicating regularity at the magnetic axis in the case of cylindrical or toroidal plasmas. Periodicity of all three components of $\boldsymbol{\xi}$ in the tangential directions is tacitly assumed. This remarkable reduction is due to the powerful representation by means of the displacement vector $\boldsymbol{\xi}$ for equilibria with nested flux surfaces (remaining valid for toroidal plasmas rotating in both the toroidal and the poloidal direction), which permits elimination of the tangential components. The innermost and outermost flux surfaces (labeled by $x_{\ell}$ and $x_{\mathrm{r}}$, respectively) are assumed to fit with the inner and outer wall, which may be real or fictitious (e.g., just indicating the range of finite perturbations of an accretion disk). Extension to BCs on exterior vacuum variables (to describe external kink modes) is considered in Sec. III.

Although several papers on the spectral problem of stationary equilibria have appeared (e.g., the early papers by Lynden-Bell and Ostriker, ${ }^{9}$ Spies, ${ }^{10}$ Hameiri, ${ }^{11,12}$ Bondeson et al.,${ }^{13}$ etc.), their present total number is negligible compared to the vast number of papers on the spectra of static equilibria. Consequently, the static 'intuition' has not been replaced by the more appropriate intuition for moving plasmas that might be derived from studies of the stationary problem. One reason may be the advent of high-resolution supercomputing, permitting the numerical solution of the original nonlinear MHD equations (1)-(4), or the linearized spectral equations (5), as a special case of more general fluid equations with dissipation, providing solutions for the dynamics of plasmas in the actual toroidal geometry of tokamaks while accounting for a multitude of physical effects (as in, e.g., the numerical program $\mathrm{CASTOR}^{14}$ and later derivatives). A more intrinsic reason is probably the fact that the Frieman-Rotenberg spectral problem widely, and inappropriately, has been classified as non self-adjoint and, therefore, hard to solve by standard methods. However, self-adjointness of the generalized force operator $\mathbf{G}$ was proved right away in Ref. 6, and the mentioned even stronger property of 'super-self-adjointness' trivially holds for the Doppler-Coriolis shift operator $U$. Clearly, the appearance of the latter operator, though crucial for the physics, can not be a valid reason for this misunderstanding.

A more intrinsic reason of why the eigenvalue problem (7)-(10) presents such an obstacle to solution is that it is a nonlinear eigenvalue problem, so that the eigenvalues are essentially complex, whereas the eigenfunctions are eigenfunctions of neither $\mathbf{G}$ nor $U$. This is to be contrasted to the static case, which is formally also nonlinear since $\omega$ appears squared. This is fundamental (since it allows for instabilities), but trivial: the complex eigenvalues are 
restricted to lie along the real and imaginary axes only. However, the essential physical properties of self-adjointness and energy conservation, which make the static case so robust, still hold for the stationary case! We will base our method on precisely those properties and demonstrate how to exploit the two quadratic forms $W$ and $V$ to obtain the complete spectrum of complex eigenvalues of stationary plasma equilibria.

Introducing a Hilbert space with an inner product definition and a finite norm,

$$
\langle\boldsymbol{\eta}, \boldsymbol{\xi}\rangle \equiv \frac{1}{2} \int \rho \boldsymbol{\xi}^{*} \cdot \boldsymbol{\eta} d V, \quad I[\boldsymbol{\xi}] \equiv\|\boldsymbol{\xi}\|^{2} \equiv\langle\boldsymbol{\xi}, \boldsymbol{\xi}\rangle<\infty,
$$

self-adjointness of the operators $\mathbf{G}$ and $U$ is expressed by

$$
\left\langle\boldsymbol{\eta}, \rho^{-1} \mathbf{G}(\boldsymbol{\xi})\right\rangle=\left\langle\rho^{-1} \mathbf{G}(\boldsymbol{\eta}), \boldsymbol{\xi}\right\rangle, \quad\langle\boldsymbol{\eta}, U \boldsymbol{\xi}\rangle=\langle U \boldsymbol{\eta}, \boldsymbol{\xi}\rangle,
$$

and reality of the associated quadratic forms (11) follows by identifying $\boldsymbol{\eta}$ with $\boldsymbol{\xi}^{*}$. The eigenvalue problem (7)-(10) then yields a quadratic equation for the eigenvalue $\omega$ :

$$
\omega^{2}-2 \bar{V} \omega-\bar{W}=0, \quad \bar{V} \equiv V / I, \quad \bar{W} \equiv W / I,
$$

where the rescaled functions $\bar{W}$ and $\bar{V}$ represent the solution averages (or expectation values) of the operators $-\rho^{-1} \mathbf{G}$ and $U$. The 'solution' $\omega=\sigma+\mathrm{i} \nu$ of this quadratic equation provides a clear split in the wave frequencies $\sigma$ and the instability growth rates $\nu$ of the modes:

$$
\omega=\bar{V} \pm\left(\bar{W}+\bar{V}^{2}\right)^{1 / 2} \Rightarrow \begin{cases}\sigma=\bar{V} \pm\left(\bar{W}+\bar{V}^{2}\right)^{1 / 2}, \quad \nu=0 & \text { (stable waves) } \\ \sigma=\bar{V}, \quad \nu= \pm\left(-\bar{W}-\bar{V}^{2}\right)^{1 / 2} & \text { (instabilities) }\end{cases}
$$

Note that each unstable eigenvalue (and its complex conjugate) is located at a distance $|\omega|=\sqrt{-\bar{W}}$ from the origin and at the horizontal distance $\sigma=\bar{V}$ from the imaginary axis (see Fig. 1). However, as intuitive as they may appear, these expressions are no more than just a posteriori properties of the implicit functions $\bar{W}(\sigma, \nu)$ and $\bar{V}(\sigma, \nu)$ that should hold for the solutions of the boundary value problem (BVP) (7)-(10), once they are obtained. The key question is: how to turn them into a procedure to actually obtain those solutions?

\section{B. The complementary energy}

At this point, it is to be realized that the solution of a nonlinear eigenvalue problem is hard, not so much because it is difficult to solve the pertinent ordinary or partial differential equations (quite effective and accurate numerical methods exist), but to systematically constrain the complex parameter $\omega$ in the associated iterative procedure that eventually produces the eigenvalue. The excellent numerical methods that exist for linear eigenvalue problems, e.g. the Jacobi-Davidson algorithm ${ }^{15}$ (implemented in our numerical program Phoenix for rotating toroidal plasmas ${ }^{16,17}$ ), do not exploit any physical properties (as they should for an effective numerical algorithm to be applicable to a wide range of scientific and technological problems). Here, we will follow precisely the opposite approach: we will systematically exploit the self-adjointness of the operators $\mathbf{G}$ and $U$, associated with the physical effects of the conservation of energy and of the Doppler-Coriolis shift of moving plasmas, in order to exploit as much physical intuition as possible on this problem. 
To that end, let us assume that we have an effective solver at our disposal for the solution of the set of differential equations (7). Just dropping the right $\mathrm{BC}$ (10), but imposing the left one, these equations can then be solved for arbitrary complex values of the parameter $\omega$. This provides solutions $\boldsymbol{\xi}^{\ell}(x ; \omega)$, which we will call the left solutions. (Recall that $x$ labels the flux surfaces and the other two coordinates are suppressed.) Obviously, integrating from left to right, the right $\mathrm{BC}$ will not be satisfied in general. The question is: what is the physical significance of this defect? To answer it, we turn to the proof of self-adjointness of the operator $\mathbf{G}$ (see, e.g., Sec. 12.2.3 of Ref. 3,), as expressed by the first inner product equality (13). That proof consists of demonstrating that both the left and the right hand side of that equality can be converted into a symmetric expression and a divergence, which is then transformed into two surface integrals over the bounding surfaces $S_{\text {left }}$ and $S_{\text {right }}$. Those surface integrals would vanish if both BCs (9) and (10) were imposed, so that only a symmetric expression would remain, which would complete the proof. With an open right boundary, this is no longer the case:

$$
\int \boldsymbol{\eta} \cdot \mathbf{G}(\boldsymbol{\xi}) d V=\int[\text { symm. in } \boldsymbol{\xi} \text { and } \boldsymbol{\eta}] d V-\int \eta \Pi(\boldsymbol{\xi}) d S_{\text {right }},
$$

since the integral over the right surface no longer vanishes, so that the operator $\mathbf{G}$ now becomes essentially non-self-adjoint. Here, $\Pi \equiv-\gamma p \nabla \cdot \boldsymbol{\xi}-\boldsymbol{\xi} \cdot \nabla p+\mathbf{B} \cdot \mathbf{Q}$ indicates the Eulerian total pressure perturbation, where $\mathbf{Q} \equiv \nabla \times(\boldsymbol{\xi} \times \mathbf{B})$ is the magnetic field perturbation, and $\boldsymbol{\xi}$ and $\boldsymbol{\eta}$ are understood to be left solutions (the superscript $\ell$ will usually be suppressed). Substituting $\boldsymbol{\eta}=\boldsymbol{\xi}^{*}$ into Eq. (16) and multiplying with $-\frac{1}{2}$ yields an expression for the energy. The non-self-adjointness of $\mathbf{G}$ then turns out to be associated with the fact that the energy of an open system is complex:

$$
W \equiv-\frac{1}{2} \int \boldsymbol{\xi}^{*} \cdot \mathbf{G}(\boldsymbol{\xi}) d V=W_{\mathrm{p}}+W_{\text {com }}
$$

Here, the symmetric part is the usual volume integral for the plasma energy $W_{\mathrm{p}}$, which is real, whereas the non-symmetric surface integral, to be called the complementary energy,

$$
W_{\text {com }}^{\ell}\left[\boldsymbol{\xi}^{\ell}\left(x_{\mathrm{r}} ; \omega\right)\right] \equiv \frac{1}{2} \int \xi^{\ell *} \Pi\left(\boldsymbol{\xi}^{\ell}\right) d S_{\text {right }}
$$

is complex. This is the amount of energy, to be provided in addition to the plasma energy, to maintain the time dependence $\exp (-\mathrm{i} \omega t)$ of the perturbations of the system. In Sec. II C, we will discuss how this expression is related to the real (in-phase) and imaginary (out-of-phase) parts of the complementary power.

The expression (18) for the complementary energy will be used for the actual solution of the closed BVP (7)-(10), including the right BC (10), as follows. We claim (and will prove in Sec. IID) that obtaining the eigenvalues, i.e. satisfying the latter BC to close off the system, is equivalent to concurrently solving two algebraic equations:

$$
W_{\text {com }, 2} \equiv \operatorname{Im}\left(W_{\text {com }}\right)=\frac{1}{2} \int\left(\xi_{1} \Pi_{2}-\xi_{2} \Pi_{1}\right) d S_{\text {right }}=0 \quad \Rightarrow \text { solution path }
$$

and

$$
W_{\mathrm{com}, 1} \equiv \operatorname{Re}\left(W_{\mathrm{com}}\right)=\frac{1}{2} \int\left(\xi_{1} \Pi_{1}+\xi_{2} \Pi_{2}\right) d S_{\text {right }}=0 \Rightarrow \text { conjugate path } .
$$


Here, the subscripts 1 and 2 indicate the real and imaginary part of the complex function. The mentioned two paths consist of all solutions of the respective equations (19) and (20), but they need to be obtained only in a strip of the $\omega$-plane, as will become clear below. The eigenvalues are situated on the intersections of the two paths, so that the complex spectrum of stationary plasmas is found by determining the common zeros of the functions $W_{\text {com }, 2}\left\{\boldsymbol{\xi}^{\ell}\left(x_{\mathrm{r}} ; \omega\right)\right\}$ and $W_{\text {com }, 1}\left\{\boldsymbol{\xi}^{\ell}\left(x_{\mathrm{r}} ; \omega\right)\right\}$. Although these expressions are highly implicit, since they involve the left solutions of the open BVP (7)-(9) for the arbitrarily chosen values of the parameter $\omega$, this is a straightforward numerical task. Evaluation of the two surface integrals just involves substitution of the right boundary values of the left solutions, which should be available in whatever numerical scheme is used.

Figure 1 is an elementary illustration of the two paths and eigenvalues for the example of an internal kink mode of a cylindrical plasma with a force-free magnetic field. ${ }^{18}$ This example is for a plasma moving at a constant speed $v_{z}$, so that the picture is identical to that of the corresponding static plasma, except that it is Doppler shifted by the constant amount $\sigma=k v_{z}$. Notwithstanding its simplicity, it illustrates the essence of what we will call the Spectral Web: the spectrum of complex eigenvalues of a stationary plasma is obtained together with the connecting structure of the solution path and the conjugate path. Concerning the terminology: the term "solution path" was already introduced in Ref. 24, the new concept of "conjugate path" is introduced here, not in the sense of complex conjugate but in the sense of conjugate to the solution path (somewhat like the momentum conjugate to a generalized coordinate in classical mechanics).

The peculiar order of the two equations (19) and (20) is chosen to stress that the solution path is the more important one. To underline this, consider how the quadratic equation (14) and its 'solutions' (15), which are valid for eigenvalues only, are modified for arbitrary values of $\omega$ by the two components $W_{\text {com, } 1}$ and $W_{\text {com, } 2}$ of the complementary energy:

$$
\omega^{2}-2 \bar{V} \omega-\bar{W}_{\mathrm{p}}=\bar{W}_{\mathrm{com}} \Rightarrow\left\{\begin{array}{l}
\sigma=\bar{V} \pm\left(\bar{W}_{\mathrm{p}}+\bar{V}^{2}+\bar{W}_{\mathrm{com}, 1}\right)^{1.2}, \quad \nu=0 \\
\sigma=\bar{V}+\frac{\bar{W}_{\mathrm{com}, 2}}{2 \nu}, \quad|\omega|=\left(-\bar{W}_{\mathrm{p}}-\bar{W}_{\mathrm{com}, 1}+\frac{\sigma}{\nu} \bar{W}_{\mathrm{com}, 2}\right)^{1 / 2}
\end{array}\right.
$$

The first line shows that the solution path for the stable waves is just the horizontal axis $\nu=0$, where $\bar{W}_{\text {com1 }}$ determines the distance to the real eigenvalues. The expression for $\sigma$ on the second line, upon substitution of $\bar{W}_{\text {com,2 }}=0$, provides an alternative expression for the solution path of the instabilities:

$$
\sigma-\bar{V}\left[\boldsymbol{\xi}^{\ell}(x ; \omega)\right] \equiv \sigma-\int \rho \boldsymbol{\xi}^{\ell *} \cdot U \boldsymbol{\xi}^{\ell} d V / \int \rho\left|\boldsymbol{\xi}^{\ell}\right|^{2} d V=0 \quad \Rightarrow \text { solution path } .
$$

This shows the physical significance of the solution path: on it, the solution averaged Doppler-Coriolis shifted frequency vanishes, as required for instabilities. In a sense, the solution path is the counterpart for stationary plasmas of the imaginary axis on which the instabilities of static plasmas are restricted to lie. Thus, for plane waves in a homogeneous plasma (never mind that instabilities will be absent in that case), the solution path is given by the constant Doppler shift $\sigma=\bar{V}=\mathbf{k} \cdot \mathbf{v}$, which is just a line parallel to the imaginary axis (like in Fig. 1). This does not imply that it remains a simple, or even a single, curve for arbitrary flows (as we will see). However, it does suggest that the solution path and, hence, all unstable eigenvalues, are restricted to lie in a strip of the complex $\omega$-plane,

$$
\sigma_{\min } \leq \sigma \leq \sigma_{\max },
$$


where $\sigma_{\min }$ and $\sigma_{\max }$ are (loosely speaking) related to the minima and maxima of $\mathbf{k} \cdot \mathbf{v}$ and (exactly) determined by the solutions of Eq. (22). For the actual computation of the solution path, the equivalent but simpler expression (19) is to be preferred though since it is a surface integral rather than a volume integral.

Figure 2 shows the Spectral Web of the force-free magnetic field equilibrium, already exploited in Fig. 1 for a trivial flow field, but now subjected to a genuine flow profile that is maximum on axis and vanishes at the wall. The solution path is split in two curves, each containing an eigenvalue; instead of one there are two instabilities in this case! We will continue the study of this intriguing configuration in the accompanying Paper II.

\section{Complementary power and energy conservation}

The Spectral Web construction by means of the complementary energy $W_{\text {com }}^{\ell}$, defined in Eq. (18), is closely related to the generalization of the energy conservation law for the open boundary problem. To derive this law, in addition to the potential energy $W$, defined in Eq. (17), we need to define the kinetic energy,

$$
K \equiv \frac{1}{2} \int \rho\left|\frac{\partial \boldsymbol{\xi}}{\partial t}\right|^{2} d V=|\omega|^{2} I
$$

and the complementary power corresponding to the complementary energy:

$$
P_{\mathrm{com}}^{\ell} \equiv-\int \frac{\partial \xi^{\ell *}}{\partial t} \Pi\left(\boldsymbol{\xi}^{\ell}\right) d S_{\mathrm{right}}=-2 \mathrm{i} \omega^{*} W_{\mathrm{com}}^{\ell}
$$

This is the power to be injected into or extracted from the plasma, through the boundary $S_{\text {right }}$, in order to maintain the prescribed time dependence $\xi \sim \exp (-\mathrm{i} \omega t)$ of the perturbations. For definiteness, one could think of an active system of antennas coupled to the plasma, or even of some 'magical' mechanical system controlling the motion of the complete plasma boundary. However, there is no need to actually specify such a system because, for our purpose, the complementary power is just an auxiliary expression in the calculation of the eigenvalues, which has to vanish in the end.

We now construct the inner product of the time dependent (left) equation (7) with $\partial \boldsymbol{\xi}^{*} / \partial t$, add the complex conjugate equation, and apply the self-adjointness relation (16) to the vector fields $\boldsymbol{\xi}^{*}$ and $\partial \boldsymbol{\xi} / \partial t$. This yields an expression for the rate of change of the total energy, which appears to be complex:

$$
\begin{aligned}
\frac{d}{d t}(W+K) & =-\frac{1}{2} \int\left[\boldsymbol{\xi}^{*} \cdot \mathbf{G}\left(\frac{\partial \boldsymbol{\xi}}{\partial t}\right)-\frac{\partial \boldsymbol{\xi}}{\partial t} \cdot \mathbf{G}\left(\boldsymbol{\xi}^{*}\right)\right] d V \\
& =\frac{1}{2} \int\left[\xi^{*} \Pi\left(\frac{\partial \boldsymbol{\xi}}{\partial t}\right)-\frac{\partial \xi}{\partial t} \Pi\left(\boldsymbol{\xi}^{*}\right)\right] d S_{\text {right }}
\end{aligned}
$$

However, extracting the asymmetric part $W_{\text {com }}$ from $W$ by means Eqs. (17) and (18) and moving it to the RHS, we obtain a real expression, which we will call the complemented energy conservation equation:

$$
\begin{gathered}
\frac{d}{d t}\left(W_{\mathrm{p}}+K\right)=-\frac{1}{2} \int\left(\frac{\partial \xi^{*}}{\partial t} \Pi+\frac{\partial \xi}{\partial t} \Pi^{*}\right) d S_{\text {right }} \equiv \operatorname{Re}\left(P_{\text {com }}\right) \\
\Rightarrow \quad 2 \nu\left(W_{\mathrm{p}}+K\right)=\operatorname{Re}\left(P_{\text {com }}\right) \equiv-2 \nu W_{\text {com }, 1}+2 \sigma W_{\text {com }, 2}
\end{gathered}
$$


The last line is due to the time dependence $\exp (2 \nu t)$ of the quadratic forms. Note that the dependence on the solution average $V$ of the operator $U$ has been annihilated here because of the super-self-adjointness of that operator.

Of course, since the complemented energy conservation equation is just a real expression, it cannot describe the full dynamics of the system contained in the complex quadratic (21). This quadratic may be cast in the form

$$
\omega K-2 \omega \omega^{*} V-\omega^{*} W_{\mathrm{p}}=\frac{1}{2} \mathrm{i} P_{\text {com }},
$$

where the real part is consistent with the complemented energy conservation equation (27). The imaginary part yields the missing information on the distribution of the different kinds of energy during the oscillations:

$$
2 \sigma\left(W_{\mathrm{p}}-K\right)+4|\omega|^{2} V=\operatorname{Im}\left(P_{\mathrm{com}}\right) \equiv-2 \sigma W_{\mathrm{com}, 1}-2 \nu W_{\text {com }, 2} .
$$

Hence, the solution average $V$ turns up again in the complex conjugate expression of the complementary power. Together, the conjugate expressions (27) and (29) show that the system needs to be provided with the power $P_{\text {com }, 1}$, in-phase with the oscillations, and with the power $P_{\text {com,2 }}$, out-of-phase with the oscillations, to be maintained at the prescribed time dependence. Eigenvalues are obtained when both $W_{\text {com,1 }}$ and $W_{\text {com,2 }}$ vanish, consistent with Eqs. (19) and (20), i.e. when the system is closed.

\section{Application of the Solution Path method}

As already noted, there is no need to actually specify a physical system providing the complementary power since the associated energies $W_{\text {com,1 }}$ and $W_{\text {com,2 }}$ may be considered to be mere auxiliary quantities that eventually have to vanish in the numerical procedure. This may be effected in two ways, as detailed below, viz. by blindly contour plotting the full Spectral Web or by iteratively solving the open BVP along the solution path. For the latter procedure, the concept of alternator, introduced in Ref. 24, is instrumental:

$$
R^{\ell} \equiv\left(\xi^{\ell} / \Pi^{\ell}\right)_{x_{\mathrm{r}}}
$$

In general, this quantity is complex but it is real along the solution path and purely imaginary along the conjugate path. Because of the right BC (10), it vanishes for eigenvalues (indicated by dots in Fig. 2) and blows up for zeros of $\Pi_{x_{\mathrm{r}}}^{\ell}$ (indicated by crosses in Fig. 2). In general, these zeros alternate along the solution path, and also along the conjugate path, as demonstrated by Fig. 2. In the Spectral Web method, a related redundancy occurs. In the expression (18) for the complementary energy, the variables $\xi^{\ell}$ and $\Pi^{\ell}$ appear on an equal footing so that genuine eigenvalues, for $\left|\xi_{x_{\mathrm{r}}}^{\ell}\right|=0$, and spurious solutions, for $\left|\Pi_{x_{\mathrm{r}}}^{\ell}\right|=0$, are found. The latter ones are easily eliminated by applying the subsidiary condition

$$
\left|\Pi^{\ell}\left(x_{\mathrm{r}}\right)\right| \neq 0,
$$

i.e. the left expression should not be smaller than some pre-chosen smallness parameter. These false solutions are actually genuine eigenvalues of an associated BVP, where the total pressure perturbation (instead of the displacement) vanishes at the boundary.

The numerical implementation of the Spectral Web method involves two stages, which may be executed independently: 
(a) Contour plotting of the full Spectral Web by solving the solution and conjugate path equations (19) and (20) in the strip (23) of the complex $\omega$-plane where the unstable eigenvalues are restricted to lie according to the solution averaged Doppler-Coriolis expression (22). Since, for different values of $\omega$, those solutions are completely independent of each other, this stage of computing is trivially parallel.

(b) Iterative eigenvalue search by, first, constructing the solution path from Eq. (19) and, next, finding the eigenvalue(s) and associated eigenfunctions on that path by determining the zeros of the alternator. This may be restricted to a rectangle $\left\{\sigma_{1} \leq \sigma \leq \sigma_{2}, \nu_{1} \leq \nu \leq \nu_{2}\right\}$ of the $\omega$-plane where one expects the eigenvalue(s) to lie from the contour plot. The iteration is extremely fast because of an oscillation theorem, stated below, according to which the alternator is monotonic along the solution path as well as along the conjugate path.

All explicit spectra shown in this, and the companion Paper II, have been obtained with either one and usually both of these methods.

For a one-dimensional system (plane slab or cylinder), the Frieman-Rotenberg spectral equation (7) may be transformed into a system of two ODEs for the components of the two-vector $\mathbf{X} \equiv\left(\xi^{\ell}, \Pi^{\ell}\right)^{\mathrm{T}}$ :

$$
\frac{d \mathbf{X}}{d x}=\widehat{\mathbf{K}} \cdot \mathbf{X}
$$

For solutions satisfying the left BC (9), satisfaction of the right BC (10) then occurs when the complementary energy (18) vanishes, and vice versa. For multi-dimensional systems, that reverse statement is not obvious. For example, for an axi-symmetric toroidal plasma with unit flux label $\psi$, poloidal angle $\vartheta$, and toroidal angle $\varphi$, the Fourier representation of the radial displacement for given toroidal wave number $n$ is given by

$$
\xi^{\ell}(\psi, \vartheta, \varphi)=\sum_{m} \xi_{m}^{\ell}(\psi) \mathrm{e}^{\mathrm{i}(m \vartheta+n \varphi)}
$$

so that the mentioned equivalence would read:

$$
\xi_{m}^{\ell}(1)=0 \quad(\text { all } m) \quad \Longleftrightarrow \quad W_{\text {com }}^{\ell}=\frac{1}{2} S_{\text {right }} \sum_{m} \xi_{m}^{\ell *}(1) \Pi_{m}^{\ell}(1)=0 .
$$

However, there are infinitely many boundary conditions on the left versus just one complex condition on $W_{\text {com }}^{\ell}$ on the right! For a cylindrical plasma, there is no problem since all harmonics decouple and the condition $W_{\text {com }}^{\ell}=0$ applies to each harmonic separately. Clearly, for toroidal plasmas, an amendment is in order. This is provided by, first, exploiting the general property of the linearized ideal MHD equations that they may be transformed into a system of two first order differential equations with respect to the normal derivative for the two unknowns $\xi$ and $\Pi$, and four tangential differential equations for the remaining variables. ${ }^{19,20}$ Next, by establishing the left arrow of the correspondence (34) in the Fourier representation of this system, by selecting a single variable that controls a complete Fourier package of modes, inspired by a completely different variable reduction in the matrix-shooting method developed by Freidberg and Hewitt ${ }^{21}$ for the problem of computing the resistive modes in a cylindrical equilibrium.

First, we again introduce the two-vector $\mathbf{X} \equiv\left(\xi^{\ell}, \Pi^{\ell}\right)^{\mathrm{T}}$ for the radial variables, and a four-vector $\mathbf{Y} \equiv\left(\phi^{1}, \phi^{2}, \phi^{3}, \phi^{4}\right)^{\mathrm{T}}$ for the tangential variables, all functions of $\psi$ and $\vartheta$. Furthermore, we introduce the $(2 \times 2,2 \times 4,4 \times 4$ and $4 \times 2)$ matrices $\mathbf{K}, \mathbf{L}, \mathbf{M}$ and $\mathbf{N}$ with 
elements that are first order differential operators in $\vartheta$ and also functions of $\psi$ and $\vartheta$. The system can then be written as

$$
\begin{array}{ll}
\frac{\partial \mathbf{X}}{\partial \psi}=\mathbf{K} \cdot \mathbf{X}+\mathbf{L} \cdot \mathbf{Y} & \Rightarrow \frac{\partial \mathbf{X}_{m}}{\partial \psi}=\sum_{\mu}\left(\mathbf{K}_{m \mu} \cdot \mathbf{X}_{\mu}+\mathbf{L}_{m \mu} \cdot \mathbf{Y}_{\mu}\right) \\
\mathbf{M} \cdot \mathbf{Y}=\mathbf{N} \cdot \mathbf{X} & \Rightarrow \sum_{\mu} \mathbf{M}_{m \mu} \cdot \mathbf{Y}_{\mu}=\sum_{\mu} \mathbf{N}_{m \mu} \cdot \mathbf{X}_{\mu},
\end{array}
$$

where the Fourier transformed equations are given on the right. For frequencies outside the continua, the tangential equations (36) may be inverted. Truncating the Fourier representation to $M$ harmonics, this gives a transformed system of $2 M$ first order ODEs for the determination of the $2 M$ radial variables $\left\{\mathbf{X}_{m}\right\} \equiv\left\{\xi_{m}^{\ell}(\psi), \Pi_{m}^{\ell}(\psi)\right\}$ :

$$
\frac{\partial \mathbf{X}_{m}}{\partial \psi}=\sum_{\mu} \widehat{\mathbf{K}}_{m \mu} \cdot \mathbf{X}_{\mu}, \quad \widehat{\mathbf{K}}_{m \mu} \equiv \mathbf{K}_{m \mu}+\sum_{\lambda} \sum_{\nu} \mathbf{L}_{m \lambda} \cdot\left(\mathbf{M}^{-1}\right)_{\lambda \nu} \cdot \mathbf{N}_{\nu \mu}
$$

Finite element, or finite difference, discretization on $N+1$ radial grid points $\psi=\psi_{i}$ (with $i=0,1, \ldots N)$ yields $2 M N$ algebraic equations for the determination of $2 M N$ variables from the $2 M(N+1)$ variable set $\left\{\xi_{m, i}^{\ell}, \Pi_{m, i}^{\ell}\right\}$. Fourier representing the BCs (9) and (10),

$$
\xi_{m, 0}^{\ell}=0 \quad \text { ('left' BCs, all } m \text { ), } \quad \xi_{m, N}^{\ell}=0 \quad \text { ('right' BCs, all } m \text { ), }
$$

fixes $2 M$ variables so that this leaves $2 M N$ homogeneous equations for the remaining $2 M N$ variables. Vanishing of the determinant yields the $M$ eigenvalues and associated eigenfunctions: this discretized version of the original two-sided BVP (7)-(10) is consistent.

Next, we open the right boundary by admitting a particular Fourier component with finite amplitude there, say for $m=m_{0}$, by imposing the following open BC's:

$$
\xi_{m, 0}^{\ell}=0 \quad(\text { all } m), \quad \xi_{m, N}^{\ell}=0 \quad\left(\text { all } m \text { except } m=m_{0}\right), \quad \xi_{m_{0}, N}^{\ell} \neq 0 .
$$

Hence, the same number of $2 M N$ algebraic equations is obtained and the only difference with the above eigenvalue problem is that the additional variable $\xi_{m_{0}, N}^{\ell}$ is moved to the RHS so that the system is now inhomogeneous, admitting a solution of the open BVP for any value of $\omega$. In this way, the problem has become very similar to that of the onedimensional equilibrium, where only a single Fourier component occurs. Of course, due to poloidal mode coupling, all the sideband harmonics of the principal harmonic $m=m_{0}$ will have finite amplitudes in the interior, but they are determined by the amplitude of that principal harmonic at the right boundary. In practice, instead of opening the boundary value problem by moving $\xi_{m_{0}, N}^{\ell}$ to the RHS of the algebraic system, it is expedient to exploit the corresponding variable $\Pi_{m_{0}, 0}^{\ell}$ for that purpose, so that the solutions may be normalized by imposing the additional left $\mathrm{BC} \Pi_{m_{0}, 0}^{\ell}=1$ (and letting $\xi_{m_{0}, 0}^{\ell}$ be determined by the algebraic system). In this manner, the Spectral Web method for toroidal systems has become similar to that for one-dimensional systems where, in the absence of degeneracy, $M$ solutions occur (one for each choice of $m_{0}$ ), so that only the contribution of that single harmonic will survive in the sum (34) for $W_{\mathrm{com}}^{\ell}$. Those solutions will be situated at very different locations in the complex $\omega$-plane because of the different Doppler-Coriolis ranges (23). Finally, it is important to note that the reduction (37) to a system of $M$ ODEs is used here only for the purpose of illustrating that the Spectral Web method is consistent with respect to the 
number of degrees of freedom, not to prescribe the numerical method. In fact, any toroidal spectral code can be converted to the new method by just relaxing the boundary conditions to the above ones, and calculating the associated complementary energy.

For the computation of the eigenfrequencies of waves and instabilities, monotonicity properties are extremely important. The prototype monotonicity is that of the Sturm-Liouville equation, connecting the eigenvalues with the number of nodes of the eigenfunctions. For static equilibria, a Sturm-Liouville kind of oscillation theorem could be proved ${ }^{22}$, because the spectra are actually real in terms of $\omega^{2}$. They are also real for the stable part of the spectrum of stationary equilibria ${ }^{23}$. It was generally assumed that generalization to the complex part of the spectrum is not possible since there is no unique way of counting nodes for complex functions. However, the previous preliminary papers ${ }^{24}$ with the present completion of the Spectral Web method has changed that, because the solution and conjugate paths effectively guide the solutions to the eigenvalues along curves in the complex $\omega$-plane. The alternator then provides the necessary alternative for counting nodes. This leads to the Complex Oscillation Theorem for instabilities:

The complex eigenvalues of a stationary equilibrium are situated on the intersections of the solution and conjugate paths where the complementary energy $W_{\mathrm{com}}^{\ell} \equiv \frac{1}{2} \int \xi^{\ell *} \Pi^{\ell} d S_{\text {right }}$ vanishes, while $\Pi^{\ell}\left(x_{\mathrm{r}}\right)$ should not vanish. The alternator $R^{\ell} \equiv \xi^{\ell}\left(x_{\mathrm{r}}\right) / \Pi^{\ell}\left(x_{\mathrm{r}}\right)$ is a real and monotonic function of arc length along the solution path, and purely imaginary and monotonic along the conjugate path, in between the zeros of $\Pi^{\ell}\left(x_{\mathrm{r}}\right)$ separating the eigenvalues.

The proofs of this theorem and its real counterpart (superseding the ones that appeared in Ref. 24) are given in Appendix A. For simplicity, in the formulation of the oscillation theorem and the applications of the accompanying paper II, the presentation is restricted to one-dimensional equilibria so that only one Fourier mode needs to be considered. It may be enlarged to toroidal equilibria by exploiting an alternator $R \equiv \xi_{m_{0}}(1) / \Pi_{m_{0}}(1)$ based on the non-vanishing Fourier component at the boundary, as in the above analysis.

\section{EXTENSIONS}

\section{A. Mixed representation}

So far, we have restricted the analysis to internal modes, integrating from left to right as required for left solutions. However, for some annular plasma models (widely exploited for accretion disks), it is frequently advantageous to integrate from right to left. This is the case when the solutions are localized near the inner boundary so that the magnitude of the right solution $\xi^{\mathrm{r}}\left(x_{\ell}\right)$ is considerable, while that of the left solution $\xi^{\ell}\left(x_{\mathrm{r}}\right)$ at the outer boundary may be negligible. The required expression for the complementary energy $W_{\text {com }}^{\mathrm{r}}$ of the right solutions is completely analogous to the expression (18) for the complementary energy $W_{\text {com }}^{\ell}$ of the left solutions:

$$
W_{\mathrm{com}}^{\mathrm{r}}\left[\boldsymbol{\xi}^{\mathrm{r}}\left(x_{\ell} ; \omega\right)\right] \equiv-\frac{1}{2} \int \xi^{\mathrm{r} *} \Pi\left(\boldsymbol{\xi}^{\mathrm{r}}\right) d S_{\text {left }}
$$

A minus sign appears here since the normal is pointing to the left.

In general, internal modes are localized neither on the left nor on the right boundary, they may even be negligible there, but they may have a large amplitude somewhere in the middle of the domain. For those cases, it is expedient to exploit a mixed representation of 
left and right solutions and to join them at some location $x=x_{\text {mix }}$ in the region of sizeable amplitudes by renormalizing them:

$$
\xi^{\mathrm{mix}} \equiv \xi^{\ell}\left(x_{\text {mix }}\right)=\xi^{\mathrm{r}}\left(x_{\text {mix }}\right) .
$$

The resulting expression for the mixed complementary energy then becomes

$$
W_{\mathrm{com}}^{\mathrm{mix}}\left[\boldsymbol{\xi}^{\ell}\left(x_{\mathrm{mix}} ; \omega\right) ; \boldsymbol{\xi}^{\mathrm{r}}\left(x_{\mathrm{mix}} ; \omega\right)\right] \equiv W_{\mathrm{com}}^{\ell}\left(x_{\mathrm{mix}}\right)+W_{\mathrm{com}}^{\mathrm{r}}\left(x_{\mathrm{mix}}\right)=-\frac{1}{2} \int \xi^{\mathrm{mix} *} \llbracket \Pi \rrbracket d S_{\mathrm{mix}},
$$

where the jump in a quantity $f$ is denoted by $\llbracket f \rrbracket \equiv f^{\mathrm{r}}\left(x_{\text {mix }}^{+}\right)-f^{\ell}\left(x_{\text {mix }}^{-}\right)$. The system is now considered to be open at the surface $x=x_{\text {mix }}$, where energy of the amount $W_{\text {com }}^{\text {mix }}$ is to be provided in order for the system to exhibit the time dependence $\exp (-\mathrm{i} \omega t)$. The eigenvalues are again obtained by imposing the condition that the complementary energy vanishes, i.e. that the jump in the total pressure perturbation vanishes so that the final solution becomes continuous. The arbitrary value of $x_{\text {mix }}$ may be fixed on the basis of accuracy considerations. Also note that this choice will give different Spectral Webs, although the spectrum itself (i.e. the intersections of the solution and conjugate paths) of course will be identical.

In the mixed representation, spurious solutions may occur when one of the solutions $\xi^{\ell}$ or $\xi^{\mathrm{r}}$ is oscillatory, so that it may accidentally vanish at $x=x_{\mathrm{mix}}$. Those solutions may be eliminated by applying the subsidiary condition

$$
\left|\xi\left(x_{\text {mix }}\right)\right| \neq 0 \text {. }
$$

The mixed representation will be exploited extensively since it is the most flexible one.

\section{B. External modes}

The expression (42) for the complementary energy of the mixed internal solutions also suggests how the eigenvalues of external modes in a plasma-vacuum system may be obtained. In that case, the plasma is bounded by a vacuum where the magnetic field perturbation $\hat{\mathbf{Q}}$ may be expressed in terms of the scalar potential $\hat{\Phi}$, satisfying Laplace's equation:

$$
\hat{\mathbf{Q}}=-\mathrm{i} \nabla \hat{\Phi}, \quad \nabla^{2} \hat{\Phi}=0 \quad\left(x_{\mathrm{a}} \leq x<x_{\mathrm{b}}\right) .
$$

The latter, second order, differential equation is equivalent to two first order ones for the unknowns $\hat{\Phi}$ and its normal derivative $\partial \hat{\Phi} / \partial n \equiv \mathbf{n} \cdot \nabla \hat{\Phi}$. Here, vacuum variables are distinguished from plasma variables by a hat, and the coordinate $x$ is supposed to be continued into the vacuum such that it smoothly connects at the plasma-vacuum boundary at $x=x_{\mathrm{a}}$ and also fits an outer, perfectly conducting, wall at $x=x_{\mathrm{b}}$. The 'right' solution, i.e. the normal vacuum magnetic field perturbation $\hat{Q}^{\mathrm{r}} \equiv \mathbf{n} \cdot \hat{\mathbf{Q}}^{\mathrm{r}}=-\mathrm{i} \partial \hat{\Phi}^{\mathrm{r}} / \partial n$, should satisfy the following $\mathrm{BC}$ at the wall:

$$
\hat{Q}=-\mathrm{i} \frac{\partial \hat{\Phi}}{\partial n}=0 \quad\left(x=x_{\mathrm{b}}\right) .
$$

Without a wall, i.e. when the vacuum extends to $\infty$, the same $\mathrm{BC}$ applies, with $x_{\mathrm{b}} \rightarrow \infty$.

To arrive at an expression for the complementary energy, the (left) plasma solution $\boldsymbol{\xi}^{\ell}$ is to be connected to the (right) vacuum solution $\hat{\mathbf{Q}}^{\mathrm{r}}=-\mathrm{i} \nabla \hat{\Phi}^{\mathrm{r}}$ by satisfying the first interface $B C$ [see, e.g., Ref. 2, Eq. (6.140)] on the normal components at the plasma-vacuum boundary:

$$
\mathbf{n} \cdot \nabla \times(\boldsymbol{\xi} \times \hat{\mathbf{B}}) \equiv[\hat{\mathbf{B}} \cdot \nabla-\mathbf{n} \cdot(\nabla \hat{\mathbf{B}}) \cdot \mathbf{n}] \xi=\hat{Q}=-\mathrm{i} \frac{\partial \hat{\Phi}}{\partial n} \quad\left(x=x_{\mathrm{a}}\right),
$$


where we have suppressed the superscripts. This amounts to a mere renormalization of the plasma or vacuum variables, analogous to the renormalization (41) for the internal modes. However, the second interface $B C$ [see, e.g., Ref. 2, Eq. (6.143)], relating the total pressure perturbation $\Pi(\boldsymbol{\xi})$ in the plasma to the magnetic pressure perturbation $\hat{\Pi}(\hat{\mathbf{Q}}) \equiv \hat{\mathbf{B}} \cdot \hat{\mathbf{Q}}$ in the vacuum,

$$
\Pi(\boldsymbol{\xi})-\llbracket \partial_{n} P \rrbracket \xi=\hat{\Pi}(\hat{\mathbf{Q}})=-\mathrm{i} \hat{\mathbf{B}} \cdot \nabla \hat{\Phi} \quad\left(x=x_{\mathrm{a}}\right),
$$

is not to be satisfied in the solution path method since it is to emerge only in the final solution for the EVs. The term with the discontinuity of the normal derivative $\partial_{n} P \equiv \mathbf{n} \cdot \nabla\left(p+\frac{1}{2} B^{2}\right)$ of the total pressure $P$ is absent for smooth equilibria (i.e. no skin currents, no skin vorticities, or vanishing density at the plasma-vacuum boundary).

For partial integrations over a surface $S$ (e.g. the plasma-vacuum surface), exploiting periodicity of the functions, it is expedient to utilize orthogonal coordinates $x_{1}(\equiv x), x_{2}, x_{3}$, with scale factors $h_{i} \equiv\left|\partial_{i} \mathbf{r}\right|$, where $x_{2}$ and $x_{3}$ refer to points on $S$ so that $d S=h_{2} h_{3} d x_{2} d x_{3}$. The 2-vector surface gradient of an arbitrary scalar $\Lambda$, the surface divergence of an arbitrary 2-vector $\mathbf{V}_{\mathrm{s}} \equiv\left(V_{2}, V_{3}\right)$, and the surface Laplacian of $\Lambda$ then read, respectively:

$$
\begin{aligned}
\nabla_{\mathrm{s}} \Lambda & \equiv\left(h_{2}^{-1} \partial_{2} \Lambda, h_{3}^{-1} \partial_{3} \Lambda\right), \\
\nabla_{\mathrm{s}} \cdot \mathbf{V}_{\mathrm{s}} & \equiv\left(h_{2} h_{3}\right)^{-1}\left[\partial_{2}\left(h_{3} V_{2}\right)+\partial_{3}\left(h_{2} V_{3}\right)\right], \\
\nabla_{\mathrm{s}}^{2} \Lambda & \equiv\left(h_{2} h_{3}\right)^{-1}\left[\partial_{2}\left(h_{3} h_{2}{ }^{-1} \partial_{2} \Lambda\right)+\partial_{3}\left(h_{2} h_{3}{ }^{-1} \partial_{3} \Lambda\right)\right] .
\end{aligned}
$$

In terms of this 2-vector notation, writing $\nabla \cdot \hat{\mathbf{B}}=\nabla_{\mathrm{s}} \cdot \hat{\mathbf{B}}_{\mathrm{s}}+h_{1}^{-1} \hat{\mathbf{B}}_{\mathrm{s}} \cdot \nabla_{\mathrm{s}} h_{1}=0$, the nasty term in the BC (46) may be transformed to $\mathbf{n} \cdot(\nabla \hat{\mathbf{B}}) \cdot \mathbf{n}=h_{1}^{-1} \hat{\mathbf{B}}_{\mathrm{s}} \cdot \nabla_{\mathrm{s}} h_{1}=-\nabla_{\mathrm{s}} \cdot \hat{\mathbf{B}}_{\mathrm{s}}$. The two BCs (46) and (47) then manifestly become relations between the normal and parallel gradients of the plasma and vacuum perturbations:

$$
\hat{F}^{\dagger} \xi \equiv \mathrm{i} \nabla_{\mathrm{s}} \cdot\left(\hat{\mathbf{B}}_{\mathrm{s}} \xi\right)=\frac{\partial \hat{\Phi}}{\partial n} \quad\left(x=x_{\mathrm{a}}\right)
$$

and

$$
\Pi(\boldsymbol{\xi})-\llbracket \partial_{n} P \rrbracket \xi=-\mathrm{i} \hat{\mathbf{B}}_{\mathrm{s}} \cdot \nabla_{\mathrm{s}} \hat{\Phi} \equiv \hat{F} \hat{\Phi} \quad\left(x=x_{\mathrm{a}}\right),
$$

where $\hat{F}^{\dagger}$ is the adjoint of the gradient operator $\hat{F}$ parallel to the magnetic field.

To derive the appropriate expression for the complementary energy of external modes, we again exploit the self-adjointness of the operator $\mathbf{G}$, also accounting for the vacuum variables. To that end, the pre-self-adjointness relation (16) in terms of the plasma variables $\boldsymbol{\xi}$ and $\boldsymbol{\eta}$ is extended with contributions from the corresponding vacuum variables $\hat{\mathbf{Q}}$ and $\hat{\mathbf{R}}$, where judicious integration by parts, exploiting Eqs. (45) and (46) [but not Eq. (47)!], yields

$$
\begin{aligned}
\int \boldsymbol{\eta} \cdot \mathbf{G}(\boldsymbol{\xi}) d V= & \int[\text { symm. in } \boldsymbol{\xi} \text { and } \boldsymbol{\eta}] d V-\int \llbracket \partial_{n} P \rrbracket \xi \eta d S_{\mathrm{a}} \\
& -\int \hat{\mathbf{Q}} \cdot \hat{\mathbf{R}} d \hat{V}+\int \eta \llbracket \Pi+\xi \partial_{n} P \rrbracket d S_{\mathrm{a}} .
\end{aligned}
$$

Evidently, self-adjointness of the extended operator $\mathbf{G}$, i.e. symmetry in $\boldsymbol{\xi}$ and $\boldsymbol{\eta}$ as well as in $\hat{\mathbf{Q}}$ and $\hat{\mathbf{R}}$, is obtained when the composite solution $\left\{\boldsymbol{\xi}^{\ell}, \hat{\mathbf{Q}}^{\mathrm{r}}\right\}$ also satisfies the BC (47), so that the last term vanishes. This step is dropped though in the solution path method. 
Substituting $\boldsymbol{\eta}=\boldsymbol{\xi}^{*}$ and $\hat{\mathbf{R}}=\hat{\mathbf{Q}}^{*}$ in Eq. (51), and multiplying with $-\frac{1}{2}$, again yields a split of $W$ into a symmetric (real) part and the complex complementary energy:

$$
W \equiv-\frac{1}{2} \int \boldsymbol{\xi}^{*} \cdot \mathbf{G}(\boldsymbol{\xi}) d V=W_{\mathrm{sym}}+W_{\mathrm{com}}, \quad W_{\mathrm{sym}} \equiv W_{\mathrm{p}}+W_{\mathrm{s}}+W_{\mathrm{v}} .
$$

Here, $W_{\mathrm{p}}, W_{\mathrm{s}}$, and $W_{\mathrm{v}}$ are the usual expressions for the energy of the plasma, of the plasmavacuum boundary surface, and of the vacuum (see, e.g., Ref. 3, Sec. 12.2.4), corresponding to the first three terms of Eq. (51). The last term yields the expression for the complementary energy of the system, which is now considered to be open at the plasma-vacuum boundary:

$$
W_{\mathrm{com}}^{\mathrm{pv}}\left[\boldsymbol{\xi}^{\ell}\left(x_{\mathrm{a}} ; \omega\right) ; \hat{\mathbf{Q}}^{\mathrm{r}}\left(x_{\mathrm{a}} ; \omega\right)\right]=-\frac{1}{2} \int \xi^{*} \llbracket \Pi+\xi \partial_{n} P \rrbracket d S_{\mathrm{a}} .
$$

Clearly, the eventual solution for $W_{\mathrm{com}}^{\mathrm{pv}}=0$ corresponds to satisfaction of the second interface condition and, thus, yields the required eigenvalues. Similar to Eq. (43), the spurious solutions (corresponding to having a wall at $x=x_{\mathrm{a}}$ ) may be eliminated by imposing the subsidiary condition $\left|\xi\left(x_{\mathrm{a}}\right)\right| \neq 0$.

Formally, instead of joining left and right solutions at the plasma-vacuum boundary, one could join them at some surface $x_{\mathrm{c}}$ in between $x_{\mathrm{a}}$ and $x_{\mathrm{b}}$. This requires continuing $\boldsymbol{\xi}^{\ell}$ with $\hat{\mathbf{Q}}^{\ell}$ by satisfying both interface BCs (46) and (47), whereas $\hat{\mathbf{Q}}^{\mathrm{r}}$ (or $\hat{\Phi}^{\mathrm{r}}$ ) should satisfy the outer BC (45). The normal magnetic field component $\hat{Q}=-\mathrm{i} \partial \hat{\Phi} / \partial n$ is then assumed to be continuous at $x=x_{\mathrm{c}}$, but the scalar potential itself is permitted to jump there: $\llbracket \hat{\Phi} \rrbracket \equiv \hat{\Phi}^{\mathrm{r}}\left(x_{\mathrm{c}}^{+}\right)-\hat{\Phi}^{\ell}\left(x_{\mathrm{c}}^{-}\right) \neq 0$. Also introducing scalar potentials $\hat{\Lambda}^{\ell, \mathrm{r}}$ for the variables $\hat{\mathbf{R}}^{\ell, \mathrm{r}}$, the appropriate pre-self-adjointness relation, replacing Eq. (51), becomes

$$
\begin{aligned}
\int \boldsymbol{\eta} \cdot \mathbf{G}(\boldsymbol{\xi}) d V= & \int[\text { symm. in } \boldsymbol{\xi} \text { and } \boldsymbol{\eta}] d V-\int \llbracket \partial_{n} P \rrbracket \xi \eta d S_{\mathrm{a}} \\
& -\int \hat{\mathbf{Q}}^{\ell} \cdot \hat{\mathbf{R}}^{\ell} d \hat{V}^{\ell}-\int \hat{\mathbf{Q}}^{\mathrm{r}} \cdot \hat{\mathbf{R}}^{\mathrm{r}} d \hat{V}^{\mathrm{r}}-\int \frac{\partial \hat{\Lambda}}{\partial n} \llbracket \hat{\Phi} \rrbracket d S_{\mathrm{c}} .
\end{aligned}
$$

The corresponding expression for the complementary energy then reads:

$$
W_{\mathrm{com}}^{\mathrm{vv}}\left[\hat{\Phi}^{\ell}\left(x_{\mathrm{c}} ; \omega\right) ; \hat{\Phi}^{\mathrm{r}}\left(x_{\mathrm{c}} ; \omega\right)\right] \equiv \frac{1}{2} \int \frac{\partial \hat{\Phi}^{*}}{\partial n} \llbracket \hat{\Phi} \rrbracket d S_{\mathrm{c}} .
$$

Of course, for the computation of the spectrum of external modes, the expression (53) is to be preferred. However, the expression (55) provides the basis for the modification of the next section when the surface at $x=x_{\mathrm{c}}$ becomes a resistive wall. Again, the spurious solutions (corresponding to having a perfectly conducting wall at $x=x_{\mathrm{c}}$ ) may be eliminated by imposing the subsidiary condition $\left|\partial \hat{\Phi} / \partial n\left(x_{\mathrm{c}}\right)\right| \neq 0$.

Summarizing, we have derived the following complementary energy expressions:

- Eq. (18) for $W_{\text {com }}^{\ell}$ of the left internal modes, Eq. (40) for $W_{\text {com }}^{\mathrm{r}}$ of the right internal modes, and Eq. (42) for $W_{\text {com }}^{\text {mix }}$ of the mixed internal modes,

- Eq. (53) for $W_{\mathrm{com}}^{\mathrm{pv}}$ of the mixed plasma-vacuum external modes, and Eq. (55) for $W_{\mathrm{com}}^{\mathrm{vv}}$ of the mixed vacuum-vacuum external modes, to be extended to Eq. (62) below for $W_{\mathrm{com}}^{\mathrm{rw}}$ of the resistive wall modes.

Nearly all of these expressions will be exploited to construct the corresponding Spectral Webs in forthcoming papers. 


\section{Resistive wall modes}

As shown in Sec. II C, computation of the MHD spectrum of a stationary equilibrium by means of the Spectral Web is based on the complemented energy conservation equation (27) and its conjugate relation (29). This suggests that the method may be extended to the description of resistive wall modes (RWMs) ${ }^{25,26}$ if we properly account for the Ohmic dissipation rate. The latter will result in an extension of the expression (55) for the complementary energy of a plasma-vacuum system, still considered open at the intermediate vacuum surface $x=x_{\mathrm{c}}$, which now becomes the position of a resistive wall. To indicate this change, we will consistently indicate that position by $x=x_{\mathrm{rw}}$ (where $x_{\mathrm{a}}<x_{\mathrm{rw}}<x_{\mathrm{b}}$ ).

At that surface, we assume the presence of a resistive wall of infinitesimal thickness $\delta$, such that the surface resistivity $\eta^{\mathrm{s}} \equiv \lim _{\eta \rightarrow 0, \delta \rightarrow 0}(\eta / \delta)$ and the surface current density perturbation $\mathbf{j}^{\mathrm{s}} \equiv \lim _{j \rightarrow \infty, \delta \rightarrow 0}(\mathbf{j} \delta)$ remain finite. Except for the complementary energy (55) to be injected into or extracted from the vacuum, we then also need to account for the Ohmic dissipation rate in the resistive wall, which is a real expression:

$$
\begin{aligned}
D & \equiv \int \eta|\mathbf{j}|^{2} d V_{\mathrm{rw}} \equiv \int \eta^{\mathrm{s}}\left|\mathbf{j}^{\mathrm{s}}\right|^{2} d S_{\mathrm{rw}}=\int \eta^{\mathrm{s}}|\mathbf{n} \times \llbracket \hat{\mathbf{Q}} \rrbracket|^{2} d S_{\mathrm{rw}} \\
& =\int \eta^{\mathrm{s}} \llbracket \nabla_{\mathrm{s}} \hat{\Phi} \rrbracket^{2} d S_{\mathrm{rw}}=-\int \nabla_{\mathrm{s}} \cdot\left(\eta^{\mathrm{s}} \llbracket \nabla_{\mathrm{s}} \hat{\Phi}^{*} \rrbracket\right) \llbracket \hat{\Phi} \rrbracket d S_{\mathrm{rw}} .
\end{aligned}
$$

For the latter transformations, we have exploited the expressions (48). Of course, eventual self-adjointness of the extended operator $\mathbf{G}$ is now excluded, but we will show that the complemented energy conservation law again leads to an expression for the complementary energy from which the complete spectrum of RWMs may be obtained.

As in the derivation of Eq. (55) of Sec. IIIB, the left solution is composed of $\boldsymbol{\xi}^{\ell}$ on the plasma interval $\left(0 \leq x \leq x_{\mathrm{a}}\right)$, 'smoothly' connected to $\hat{\mathbf{Q}}^{\ell}$ ( or $\hat{\Phi}^{\ell}$ ) on the internal vacuum interval $\left(x_{\mathrm{a}}<x<x_{\mathrm{rw}}\right)$ by satisfying both interface BCs (46) and (47), whereas the right solution is given by $\hat{\mathbf{Q}}^{\mathrm{r}}$ (or $\hat{\Phi}^{\mathrm{r}}$ ) on the external vacuum interval $\left(x_{\mathrm{rw}}<x<x_{\mathrm{b}}\right.$ ), satisfying the $\mathrm{BC}(45)$ at the outer perfectly conducting wall at $x=x_{\mathrm{b}}($ or $\infty)$. Again, $\hat{Q}=-\mathrm{i} \partial \hat{\Phi} / \partial n$ is continuous at $x=x_{\mathrm{rw}}$, but the discontinuity of $\hat{\Phi}$ at the resistive wall now gives rise to the two expressions $W_{\mathrm{com}}^{\mathrm{vv}}$ and $D$, which both should somehow appear in the expression for the complementary energy $W_{\mathrm{com}}^{\mathrm{rw}}$ of the resistive wall problem that we are looking for.

For the eventual solutions of the eigenvalues, obtained from $W_{\mathrm{com}}^{\mathrm{rw}}=0$, the corresponding eigenfunctions should satisfy the BC expressing continuity of the normal magnetic field,

$$
\llbracket \hat{Q} \rrbracket=0 \quad \Rightarrow \quad \llbracket \frac{\partial \hat{\Phi}}{\partial n} \rrbracket=0 \quad\left(x=x_{\mathrm{rw}}\right),
$$

and the crucial resistive wall BC, resulting from the laws of Faraday and Ohm:

$$
\hat{Q}+\frac{\mathrm{i}}{\omega} \mathbf{n} \cdot \nabla \times\left(\eta^{\mathrm{s}} \mathbf{n} \times \llbracket \hat{\mathbf{Q}} \rrbracket\right)=0 \quad \Rightarrow \quad \frac{\partial \hat{\Phi}}{\partial n}+\frac{\mathrm{i}}{\omega} \nabla_{\mathrm{s}} \cdot\left(\eta^{\mathrm{s}} \llbracket \nabla_{\mathrm{s}} \hat{\Phi} \rrbracket\right)=0 \quad\left(x=x_{\mathrm{rw}}\right) .
$$

For the latter conversion, we have exploited the equalities $\nabla \cdot \hat{\mathbf{Q}}=0$ and $\llbracket \hat{Q} \rrbracket=0$, and the expressions (48). For generality, as in Refs. 27 and 28, we have assumed that the value of $\eta^{\mathrm{s}}$ is not constant along the surface $S_{\mathrm{rw}}$. In the following derivation of $W_{\mathrm{com}}^{\mathrm{rw}}$, the BC (57) is again considered as a mere renormalization of the left (or right) solution, whereas the BC (58) will not be used but emerge from the final Spectral Web construction. 
Similar to the derivation of Sec. III B, consistently integrating by parts and applying the appropriate BCs (including periodicity), we may convert the general expression for $W$, as defined in the first part of Eq. (17), into a symmetric expression and a complex integral over the surface of the resistive wall:

$$
W=W_{\mathrm{sym}}+\frac{1}{2} \int \frac{\partial \hat{\Phi}^{*}}{\partial n} \llbracket \hat{\Phi} \rrbracket d S_{\mathrm{rw}}, \quad W_{\mathrm{sym}} \equiv W_{\mathrm{p}}+W_{\mathrm{s}}+W_{\mathrm{v}}^{\ell}+W_{\mathrm{v}}^{\mathrm{r}} .
$$

Here, $W_{\mathrm{p}}$ and $W_{\mathrm{s}}$ are the plasma and surface energy integrals, already encountered in Eq. (52), but the vacuum integral is split into separate contributions $W_{\mathrm{v}}^{\ell}\left(\hat{\Phi}^{\ell}\right)$, for $x<x_{\mathrm{rw}}$, and $W_{\mathrm{v}}^{\mathrm{r}}\left(\hat{\Phi}^{\mathrm{r}}\right)$, for $x>x_{\mathrm{rw}}$, since the scalar potential $\hat{\Phi}$ is no longer continuous. The integral over the resistive wall is identical (replacing $S_{\mathrm{c}}$ by $S_{\mathrm{rw}}$ ) to the definition (55) of the complementary energy $W_{\mathrm{com}}^{\mathrm{vv}}$ for the artificially split external mode problem, which appears in the following form of the complemented energy conservation equation:

$$
\frac{d}{d t}\left(W_{\mathrm{sym}}+K\right)=\operatorname{Re}\left(P_{\mathrm{com}}^{\mathrm{vv}}\right), \quad P_{\mathrm{com}}^{\mathrm{vv}}=-2 \mathrm{i} \omega^{*} W_{\mathrm{com}}^{\mathrm{vv}} .
$$

Although this equation is also valid for the resistive wall mode problem, the expression $W_{\mathrm{com}}^{\mathrm{vv}}$ does not represent the complementary energy of the full system now since we still have to account for the resistive dissipation $D$. This is done by writing $P_{\mathrm{com}}^{\mathrm{rw}} \equiv P_{\mathrm{com}}^{\mathrm{vv}}+D$, giving the following, resistive, modification of the complemented energy conservation equation:

$$
\begin{aligned}
& \frac{d}{d t}\left(W_{\mathrm{sym}}+K\right)+D=\operatorname{Re}\left(P_{\mathrm{com}}^{\mathrm{rw}}\right), \\
& P_{\mathrm{com}}^{\mathrm{rw}} \equiv-\int\left\{\frac{\partial}{\partial t} \frac{\partial \hat{\Phi}^{*}}{\partial n}+\nabla_{\mathrm{s}} \cdot\left(\eta^{\mathrm{s}} \llbracket \nabla_{\mathrm{s}} \hat{\Phi}^{*} \rrbracket\right)\right\} \llbracket \hat{\Phi} \rrbracket d S_{\mathrm{rw}}=-2 \mathrm{i} \omega^{*} W_{\mathrm{com}}^{\mathrm{rw}} .
\end{aligned}
$$

This yields the required expression for the complementary energy of RWMs:

$$
W_{\mathrm{com}}^{\mathrm{rw}}\left[\hat{\Phi}^{\ell}\left(x_{\mathrm{rw}} ; \omega\right) ; \hat{\Phi}^{\mathrm{r}}\left(x_{\mathrm{rw}} ; \omega\right)\right] \equiv \frac{1}{2} \int\left\{\frac{\partial \hat{\Phi}^{*}}{\partial n}-\frac{\mathrm{i}}{\omega^{*}} \nabla_{\mathrm{s}} \cdot\left(\eta^{\mathrm{s}} \llbracket \nabla_{\mathrm{s}} \hat{\Phi}^{*} \rrbracket\right)\right\} \llbracket \hat{\Phi} \rrbracket d S_{\mathrm{rw}},
$$

consistent with the resistive wall BC (58). Here, the spurious solutions (corresponding to absence of the resistive wall) may be eliminated by imposing the subsidiary condition $\left|\llbracket \hat{\Phi}\left(x_{\mathrm{rw}}\right) \rrbracket\right| \neq 0$.

The full spectrum of RWMs may be obtained by constructing the Spectral Web from the two conditions that the real and the imaginary part of the expression (62) should vanish separately. This involves a relation in addition to the complementary energy equation, since that contains only real quantities. The missing information may be obtained from the original complex quadratic equation (14), which is completely general as it does not depend on any BCs. Substitution of the expression $W=W_{\text {sym }}-\frac{1}{2}\left(\mathrm{i} / \omega^{*}\right) D+W_{\text {com }}^{\mathrm{rw}}$, following from Eqs. (59) and (62), gives

$$
\omega^{2} I-2 \omega V-W_{\mathrm{sym}}+\frac{\mathrm{i}}{2 \omega^{*}} D=W_{\mathrm{com}}^{\mathrm{rw}} .
$$

Equivalently,

$$
\begin{aligned}
\omega K-2|\omega|^{2} V-\omega^{*} W_{\mathrm{sym}}+\frac{1}{2} \mathrm{i} D & =\frac{1}{2} \mathrm{i} P_{\mathrm{com}}^{\mathrm{rw}} \Rightarrow \\
2 \nu\left(W_{\mathrm{sym}}+K\right)+D & =\operatorname{Re}\left(P_{\mathrm{com}}^{\mathrm{rw}}\right) \equiv-2 \nu W_{\mathrm{com}, 1}^{\mathrm{rw}}+2 \sigma W_{\mathrm{com}, 2}^{\mathrm{rw}}, \\
2 \sigma\left(W_{\mathrm{sym}}-K\right)+4|\omega|^{2} V & =\operatorname{Im}\left(P_{\mathrm{com}}^{\mathrm{rw}}\right) \equiv-2 \sigma W_{\mathrm{com}, 1}^{\mathrm{rw}}-2 \nu W_{\mathrm{com}, 2}^{\mathrm{rw}} .
\end{aligned}
$$


where the first relation reproduces the complemented energy conservation equation (61), and the second one is the missing conjugate relation needed to complete the representation. Hence, the Spectral Web is obtained from the two components of the complex equation $P_{\mathrm{com}}^{\mathrm{rw}}=0$, or from the two components of $W_{\mathrm{com}}^{\mathrm{rw}}=0$; QED.

\section{SUMMARY}

- The new concept of Spectral Web is introduced to compute the complex frequencies of the waves and instabilities of moving plasmas, based on the self-adjointness of the generalized Frieman-Rotenberg force operator, $\mathbf{G}$, and the Doppler-Coriolis gradient operator parallel to the velocity, $U$ (Sec. II A).

- The problem is solved with an open boundary, where the complementary energy $W_{\text {com }}$ represents the amount of energy to be delivered to or extracted from the system to maintain harmonic time-dependence (Secs. II B and II C).

- The eigenvalues are connected by a system of curves in the complex $\omega$-plane, the solution path and the conjugate path (where $W_{\text {com }}$ is real or imaginary), with peculiar geometries that have to be clarified yet, but that have a deep physical significance (Sec. II B).

- The Spectral Web is obtained by straightforward contour plotting of the two paths, the complex eigenvalues within a specified rectangle of the complex $\omega$-plane are found by fast, reliable and accurate iterations (Sec. II D).

- Real and Complex Oscillation Theorems, replacing the familiar tool of counting nodes of eigenfunctions, provide an associated mechanism of mode tracking along the two paths (Sec. II D and Appendix A).

- Generalization of the method to toroidal systems is demonstrated (Sec. IID).

- The method is extended to include a resistive wall by accounting for the dissipation in such a wall (Sec. III B).

- The Spectral Web method is applied in Paper II to a multitude of the basic fundamental instabilities operating in cylindrical plasmas.

\section{Acknowledgement}

I am indebted to Rony Keppens and Stefaan Poedts for stimulating criticism and discussions and to Hugo de Blank for suggestions on terminology. 


\section{Appendix A: Proof of the Oscillation Theorems}

\section{Sequencing integrals}

Consider two neighboring points $\omega_{\alpha}$ and $\omega_{\beta}$, either on the solution path or on the conjugate path, with the corresponding left solutions $\boldsymbol{\xi}_{\alpha}$ and $\boldsymbol{\xi}_{\beta}$ of the spectral equation (7), symmetrically positioned about the point $\omega \equiv \sigma+\mathrm{i} \nu$ corresponding to the left solution $\boldsymbol{\xi}$ :

$$
\begin{array}{ccc}
\omega_{\alpha} \equiv \omega+\lambda, & \boldsymbol{\xi}_{\alpha} \equiv \boldsymbol{\xi}+\lambda \boldsymbol{\eta}, & \Pi_{\alpha} \equiv \Pi(\boldsymbol{\xi})+\lambda \Pi(\boldsymbol{\eta}), \\
\omega_{\beta} \equiv \omega-\lambda, & \boldsymbol{\xi}_{\beta} \equiv \boldsymbol{\xi}-\lambda \boldsymbol{\eta}, & \Pi_{\beta} \equiv \Pi(\boldsymbol{\xi})-\lambda \Pi(\boldsymbol{\eta}) .
\end{array}
$$

Here, $|\lambda| \ll|\omega|$, where the frequencies are made dimensionless in the usual way, and the perturbation $\lambda \boldsymbol{\eta}$ is small in norm compared to $\boldsymbol{\xi}$. From the homogeneous Frieman-Rotenberg equations (7) for $\boldsymbol{\xi}_{\alpha}$ and $\boldsymbol{\xi}_{\beta}$, one easily shows that $\boldsymbol{\eta}$ has to satisfy an inhomogeneous Frieman-Rotenberg equation,

$$
\mathbf{G}(\boldsymbol{\eta})-2 \rho \omega U \boldsymbol{\eta}+\rho \omega^{2} \boldsymbol{\eta}=2 \rho(U \boldsymbol{\xi}-\omega \boldsymbol{\xi}),
$$

so that $\boldsymbol{\eta}$ is of the same order as, and determined by, the function $\boldsymbol{\xi}$.

From the mentioned equations for $\mathbf{G}(\boldsymbol{\xi})$ and $\mathbf{G}\left(\boldsymbol{\eta}^{*}\right)$, two quadratic forms may be constructed involving two surface integrals which are perturbations of the complementary energy. They emerge from the proof of self-adjointness of the operator G:

$$
\begin{aligned}
& \frac{1}{2} \int \eta^{*} \Pi(\boldsymbol{\xi}) d S_{\text {right }} \equiv-Q\left(\boldsymbol{\xi}, \boldsymbol{\eta}^{*}\right)-\frac{1}{2} \int \boldsymbol{\eta}^{*} \cdot \mathbf{G}(\boldsymbol{\xi}) d V, \\
& \frac{1}{2} \int \xi \Pi\left(\boldsymbol{\eta}^{*}\right) d S_{\text {right }} \equiv-Q\left(\boldsymbol{\xi}, \boldsymbol{\eta}^{*}\right)-\frac{1}{2} \int \boldsymbol{\xi} \cdot \mathbf{G}\left(\boldsymbol{\eta}^{*}\right) d V .
\end{aligned}
$$

The explicit form of the quadratic expression $Q\left(\boldsymbol{\xi}, \boldsymbol{\eta}^{*}\right)$ is not needed here, only its symmetry. Subtracting and adding the two equations, multiplied with $\lambda^{*}$, yields two integrals that control the sequencing of the eigenvalues:

$$
\begin{aligned}
\Gamma & \equiv \frac{1}{2} \lambda^{*} \int\left[\eta^{*} \Pi(\boldsymbol{\xi})-\xi \Pi\left(\boldsymbol{\eta}^{*}\right)\right] d S_{\text {right }} \\
& =-\frac{1}{2} \lambda^{*} \int\left[\boldsymbol{\eta}^{*} \cdot \mathbf{G}(\boldsymbol{\xi})-\boldsymbol{\xi} \cdot \mathbf{G}\left(\boldsymbol{\eta}^{*}\right)\right] d V=2 \lambda^{*}[\mathrm{i} \nu X+(\bar{V}-\sigma) I], \\
\Delta & \equiv \frac{1}{2} \lambda^{*} \int\left[\eta^{*} \Pi(\boldsymbol{\xi})+\xi \Pi\left(\boldsymbol{\eta}^{*}\right)\right] d S_{\text {right }} \\
& =-\frac{1}{2} \lambda^{*} \int\left[\boldsymbol{\eta}^{*} \cdot \mathbf{G}(\boldsymbol{\xi})+\boldsymbol{\xi} \cdot \mathbf{G}\left(\boldsymbol{\eta}^{*}\right)\right] d V-2 \lambda^{*} Q\left(\boldsymbol{\xi}, \boldsymbol{\eta}^{*}\right)=-2 \lambda^{*} Y,
\end{aligned}
$$

where two auxiliary integrals of order unity have been introduced:

$$
\begin{aligned}
X & \equiv I-\int \rho \boldsymbol{\eta}^{*} \cdot(U \boldsymbol{\xi}-\sigma \boldsymbol{\xi}) d V \\
Y & \equiv(\bar{V}+\mathrm{i} \nu) I-\sigma X+\frac{1}{2}|\omega|^{2} \int \rho \boldsymbol{\eta}^{*} \cdot \boldsymbol{\xi} d V+Q\left(\boldsymbol{\xi}, \boldsymbol{\eta}^{*}\right) .
\end{aligned}
$$

Surprisingly little information on these integrals is needed for our purpose.

At this point, we need to distinguish between real and complex frequencies, and also split $\lambda \equiv \frac{1}{2}\left(\omega_{\alpha}-\omega_{\beta}\right) \equiv \delta+\mathrm{i} \epsilon$ to distinguish between horizontal $(\delta)$ and vertical $(\epsilon)$ components of arc length along the different paths. 


\section{Real Oscillation Theorem}

For real frequencies, transforming back to the original vectors $\boldsymbol{\xi}_{\alpha}$ and $\boldsymbol{\xi}_{\beta}$, we obtain from Eq. (A5) the following expression for the sequencing integral along the solution path (the real axis):

$$
\Gamma \equiv \frac{1}{4} \int\left(\xi_{\alpha} \Pi_{\beta}-\xi_{\beta} \Pi_{\alpha}\right) d S_{\text {right }}=2 \delta(\bar{V}-\sigma) I \approx\left(\sigma_{\alpha}-\sigma_{\beta}\right)\left[\bar{V}\left(\sigma_{\alpha}\right)-\sigma_{\alpha}\right] I .
$$

Consequently, along the real axis of the $\omega$-plane, but outside the Doppler-Coriolis indefinite range (23), where $\sigma=\{\bar{V}(x)\}$, and outside the continua and apparent singularity ranges (i.e. as long as $\Gamma$ and $\bar{V}$ are well defined), the sequencing integral $\Gamma$ is a (positive or negative) definite function of the frequency $\sigma$. This is as far as we can get without restricting the equilibrium configuration.

For one-dimensional equilibria, the expression for $\Gamma$ may be further reduced by exploiting the familiar relation $\Pi=-(N / D) \xi^{\prime}-(C / D) \xi$. The modified expressions of the coefficients $N, D$, and $C$ for stationary equilibria will be derived in paper II. Here, we only need to know that the zeros of the quartics $N$ and $D$ represent the Doppler-shifted continuous spectra and apparent singularities, respectively. Equation (A9) for a left solution $\xi_{\alpha}$ that also vanishes at the right end point then yields:

$$
\Gamma \equiv \frac{1}{4} S\left(\xi_{\alpha} \Pi_{\beta}-\xi_{\beta} \Pi_{\alpha}\right)=\frac{1}{4} S\left[\xi_{\beta} \frac{N\left(\sigma_{\alpha}\right)}{D\left(\sigma_{\alpha}\right)} \xi_{\alpha}^{\prime}\right]_{\text {right }} \approx\left(\sigma_{\beta}-\sigma_{\alpha}\right)\left[\sigma_{\alpha}-\bar{V}\left(\sigma_{\alpha}\right)\right] I \quad \text { (real axis) }
$$

Applying the Sturm-Liouville kind of reasoning to the left solution $\xi_{\beta}$ gives the required result: Outside the Doppler-Coriolis indefinite range $\{\bar{V}(x)=\sigma\}$, the continua $\{N(x)=0\}$ and the apparent singularities $\{D(x)=0\}$, the expression

$$
\frac{N(\sigma)}{[\sigma-\bar{V}(\sigma)] D(\sigma)} \begin{cases}>1 & \text { (Sturmian behavior) } \\ <1 & \text { (anti-Sturmian behavior) }\end{cases}
$$

determines whether $\xi_{\beta}$ oscillates faster, or slower, than $\xi_{\alpha}$ when $\sigma_{\beta}>\sigma_{\alpha}$; QED. This kind of reasoning is essentially restricted to real frequencies.

A much more general approach, also applicable to complex frequencies, is obtained by exploiting the alternator, $R \equiv(\xi / \Pi)_{\text {right }}$. The eigenvalues are determined by $R=0$, whereas for the spurious solutions $\Pi_{\text {right }}=0$ the alternator blows up and reverses sign to start a new stretch of monotonicity. Hence, the alternator is a powerful bookkeeper of the alternating zeros of the oscillating functions $\xi$ and $\Pi$. We will prove that $R$ is monotonic on the pieces of the solution path delimited by the zeros of $\Pi_{\text {right }}$ so that the eigenvalues (where $\xi_{\text {right }}=0$ ) are guaranteed to be situated in between.

Substitution of the alternator into the sequencing integral (A9) yields

$$
\Gamma \equiv \frac{1}{4} S\left(\xi_{\alpha} \Pi_{\beta}-\xi_{\beta} \Pi_{\alpha}\right) \approx \frac{1}{4} S\left(R_{\beta}-R_{\alpha}\right)\left|\Pi_{\alpha}\right|^{2} \approx\left(\sigma_{\beta}-\sigma_{\alpha}\right)\left[\sigma_{\alpha}-\bar{V}\left(\sigma_{\alpha}\right)\right] I \quad \text { (real axis). }
$$

Hence, excluding again the mentioned frequency ranges (where $R$ is not defined), we arrive at the final formulation of the Real Oscillation Theorem:

Along the real axis, outside the Doppler-Coriolis indefinite range, the continua and the apparent singularities, the alternator $R$ is a monotonic function of the frequency $\sigma$ in between the zeros of $\Pi_{\text {right }}$; QED. 


\section{Complex Oscillation Theorem}

For complex frequencies, also transforming back to the original vectors $\boldsymbol{\xi}_{\alpha}$ and $\boldsymbol{\xi}_{\beta}$, Eqs. (A5) and (A6) yield

$$
\begin{aligned}
\Gamma & \equiv \frac{1}{4} \operatorname{Re} \int\left(\xi_{\alpha}^{*} \Pi_{\beta}-\xi_{\beta}^{*} \Pi_{\alpha}\right) d S_{\text {right }}\left\{+\frac{1}{2} \mathrm{i} \operatorname{Im}\left(W_{\text {com }, \alpha}-W_{\text {com }, \beta}\right)\right\} \\
\Delta & \equiv \frac{1}{4} \mathrm{i} \operatorname{Im} \int\left(\xi_{\alpha}^{*} \Pi_{\beta}-\xi_{\beta}^{*} \Pi_{\alpha}\right) d S_{\text {right }}\left\{+\frac{1}{2} \operatorname{Re}\left(W_{\text {com }, \alpha}-W_{\text {com }, \beta}\right)\right\},
\end{aligned}
$$

where the expressions in curly brackets vanish on the pertinent path in the $\omega$-plane. Hence, the integral $\Gamma$ is real along the solution path, so that

$$
\Gamma=2 \nu \operatorname{sgn}(x)|\lambda||X|=\nu \operatorname{sgn}(x)|X|\left|\omega_{\alpha}-\omega_{\beta}\right|, \quad x \equiv X_{1} / \epsilon=-X_{2} / \delta,
$$

and the integral $\Delta$ is purely imaginary along the conjugate path, so that

$$
\operatorname{Im} \Delta=2 \operatorname{sgn}(y)|\lambda||Y|=\operatorname{sgn}(y)|Y|\left|\omega_{\alpha}-\omega_{\beta}\right|, \quad y \equiv Y_{1} / \epsilon=-Y_{2} / \delta .
$$

In the first expression, $|X|$ is a leading order quantity (depending on $\sigma$ and $\nu$ only) and the value of $\operatorname{sgn}(x)$ is constant (either +1 or -1 ) along the solution path. This is true as long as $X \neq 0$, so that $X_{1}$ changes sign at horizontal parts $(\epsilon=0)$ and $X_{2}$ changes sign at vertical parts $(\delta=0)$ of the solution path. However, if $X=0$ somewhere on the solution path, $\operatorname{sgn}(x)$ and hence $\Gamma$ would fail to be definite. Explicit examples in paper II will show that the solution path actually breaks up to avoid its occurrence. Consequently, excepting the case $X=0$, the sequencing integral $\Gamma$ is a real and (positive or negative) definite function of arc length along the solution path. An analogous reasoning holds for the implications of the expression for $\operatorname{Im} \Delta$ in terms of $|Y|$. Hence, excepting the case $Y=0$, the sequencing integral $\Delta$ is purely imaginary and its imaginary part is a (positive or negative) definite function of arc length along the conjugate path.

For one-dimensional equilibria, the expression for $\Gamma$ on the solution path may be reduced by means of the complex generalization of the definition for the alternator, $R \equiv(\xi / \Pi)_{\text {right }}$. However, the crucial consequence of the path expression (19) is that the alternator is real for complex frequencies on the solution path:

$$
R=R_{1}=\xi_{1} / \Pi_{1}=\xi_{2} / \Pi_{2} \quad\left(\text { at } x=x_{\mathrm{r}}\right) .
$$

Substitution into Eqs. (A13) and (A15) yields

$$
\begin{aligned}
\Gamma & =\frac{1}{4} S\left(R_{\alpha}-R_{\beta}\right)\left(\Pi_{\alpha}^{*} \Pi_{\beta}+\Pi_{\alpha} \Pi_{\beta}^{*}\right) \quad \text { (solution path) } \\
& \approx \frac{1}{4} S\left(R_{\alpha}-R_{\beta}\right)\left|\Pi_{\alpha}\right|^{2}=\nu_{\alpha} \operatorname{sgn}(x)|X|\left|\omega_{\alpha}-\omega_{\beta}\right| .
\end{aligned}
$$

As in the real case, the eigenvalues are determined by $R\left(x_{\mathrm{r}}\right)=0$, whereas for the spurious solutions $\Pi\left(x_{\mathrm{r}}\right)=0$ the alternator blows up and reverses sign to start a new stretch of monotonicity of the solution path.

Similarly, the expression for $\Delta$ on the conjugate path may also be reduced by means of the complex alternator $R$. The path expression (20) then implies that the alternator is imaginary for complex frequencies on the conjugate path:

$$
R=\mathrm{i} R_{2}=\mathrm{i} \xi_{2} / \Pi_{1}=-\mathrm{i} \xi_{1} / \Pi_{2} \quad\left(\text { at } x=x_{\mathrm{r}}\right) .
$$


Substitution into Eqs. (A14) and (A16) then yields

$$
\begin{aligned}
\operatorname{Im} \Delta & =-\frac{1}{4} S\left(R_{\alpha}-R_{\beta}\right)\left(\Pi_{\alpha}^{*} \Pi_{\beta}+\Pi_{\alpha} \Pi_{\beta}^{*}\right) \quad \text { (conjugate path) } \\
& \approx-\frac{1}{4} S\left(R_{\alpha, 2}-R_{\beta, 2}\right)\left|\Pi_{\alpha}\right|^{2}=\operatorname{sgn}(y)|Y|\left|\omega_{\alpha}-\omega_{\beta}\right| .
\end{aligned}
$$

The eigenvalues are again determined by $R\left(x_{\mathrm{r}}\right)=0$, whereas for the spurious solutions $\Pi\left(x_{\mathrm{r}}\right)=0$ the alternator blows up and reverses sign to start a new stretch of monotonicity of the conjugate path.

Consequently, we arrive at the following formulation of Complex Oscillation Theorem:

Along the solution path, in between the zeros of $\Pi$, the alternator $R$ is a real and monotonic function of arc length; along the conjugate path, in between the zeros of $\Pi$, the alternator $R$ is purely imaginary and its imaginary part $R_{2}$ is a monotonic function of arc length; QED.

Thus, the eigenvalues on either one of the paths are consistently connected by counting the number of consecutive branches of the alternator. On the real sections of the solution path, this number coincides with the number of nodes of the eigenfunction, so that a genuine generalization of the Sturm-Liouville counting procedure has been obtained.

In conclusion: Since $\Gamma\left(\boldsymbol{\xi}_{\alpha}, \boldsymbol{\xi}_{\beta}\right)$ and $\operatorname{Im} \Delta\left(\boldsymbol{\xi}_{\alpha}, \boldsymbol{\xi}_{\beta}\right)$ are definite functions on their respective paths, the progression from $\omega_{\alpha}$ to $\omega_{\beta}$, from $\omega_{\beta}$ to $\omega_{\gamma}$, etc., is essentially a monotonic one. This is one of the powerful consequences of the self-adjointness of the operators $\mathbf{G}$ and $U$, which guarantees fast convergence to the eigenvalues for iterative numerical procedures, in particular along the solution path. However, a genuine complication is due to the nonlinearity of the eigenvalue problem of stationary equilibria: the solution path and the conjugate path are usually not single curves but fragmented into many branches (hence the term 'Spectral Web'), even containing closed loops. This appears to be how occurrence of $X=0$ or $Y=0$, which would imply non-monotonicity, is avoided. A significant consequence of the monotonicity properties is that closed loops of the solution or of the conjugate path are guaranteed to contain at least one eigenvalue. The accompanying paper II gives many illustrations of these fundamental properties. 
1 J. P. Goedbloed, Phys. Plasmas, to be published.

2 J. P. Goedbloed and S. Poedts, Principles of Magnetohydrodynamics; with Applications to Laboratory and Astrophysical Plasmas (Cambridge University Press, 2004), Section 4.1.

3 J. P. Goedbloed, R. Keppens and S. Poedts, Advanced Magnetohydrodynamics; with Applications to Laboratory and Astrophysical Plasmas (Cambridge University Press, 2010), Section 12.1.

4 K. Hain, R. Lüst and A. Schlüter, Z. Naturforsch. 12a, 833 (1957).

5 I. B. Bernstein, E. A. Frieman, M. D. Kruskal and R. M. Kulsrud, Proc. Roy. Soc. (London) A244, 17 (1958).

6 E. Frieman and M. Rotenberg, Rev. Modern Physics 32, 898 (1960).

7 E. M. Barston, J. Math. Phys. 8, 523 (1960).

8 R. Keppens and T. Demaerel, Phys. Plasmas 23, 122117 (2016).

9 D. Lynden-Bell and J. P. Ostriker, Mon. Not. R. Astr. Soc. 136, 293 (1967).

10 G. O. Spies, Phys. Fluids 21, 580 (1978).

11 E. Hameiri, J. Math. Phys. 22, 2080 (1981).

12 E. Hameiri, Phys. Fluids 26, 230 (1983).

13 A. Bondeson, R. Iacono and A. H. Bhattacharjee, Phys. Fluids 30, 2167 (1987).

14 W. Kerner, J. P. Goedbloed, G. T. A. Huysmans, S. Poedts and E. Schwarz, J. Comp. Physics 142, 271 (1998).

15 G. L. G. Sleijpen and H. A. van der Vorst, SIAM J. Matrix Anal. Appl. 17, 401 (1996).

16 J. P. Goedbloed, A. J. C. Beliën, B. van der Holst and R. Keppens, Phys. Plasmas 11, 28 (2004).

17 J. W. S. Blokland, B. van der Holst, R. Keppens and J. P. Goedbloed, J. Comp. Physics 226, 509 (2007).

18 J. P. Goedbloed and H. J. L. Hagebeuk, Phys. Fluids 15, 1090 (1972).

19 E. Hameiri and J. H. Hammer, Phys. Fluids 22, 1700 (1979).

20 C. E. Kieras and J. A. Tataronis, J. Plasma Physics 28, 395 (1982).

21 J. P. Freidberg and D. W. Hewitt, J. Plasma Physics 26, 177 (1981).

22 J. P. Goedbloed and P. H. Sakanaka, Phys. Fluids 17, 908 (1974).

23 J.P. Goedbloed, A.J.C. Beliën, B. van der Holst and R. Keppens, Phys. Plasmas 11, 4332 (2004).

24 J.P. Goedbloed, Phys. Plasmas 16, 122110 (2009); Phys. Plasmas 16, 122111 (2009).

25 D. Pfirsch and H. Tasso, Nucl. Fusion 11, 259 (1971).

26 J. P. Goedbloed, D. Pfirsch and H. Tasso,, Nucl. Fusion 12, 649 (1972).

27 M. S. Chance, M. S. Chu, M. Okabayashi and A. D. Turnbull, Nucl. Fusion 42, 295 (2002).

28 M. S. Chu, M. S. Chance, A. H. Glasser and M. Okabayashi, Nucl. Fusion 43, 441 (2003). 


\section{FIGURE CAPTIONS}

Figure 1. Elementary Spectral Web consisting of solution path (red) and conjugate path (blue) with eigenvalues (black dots) at the intersections; forward and backward Alfvén continua are located on the real axis. [This example refers to the $m=1, k=1.28$ internal kink mode of a cylindrical force-free magnetic field of constant $\alpha a=8.0$, subjected to a constant Doppler shift, $\sigma=k v_{z}=0.4$.]

Figure 2. Spectral Web of the same equilibrium as in Fig. 1, but subjected to a shear velocity profile $v_{z}=1-x^{2}$. Genuine eigenvalues are indicated by a dot, false ones by a cross. 


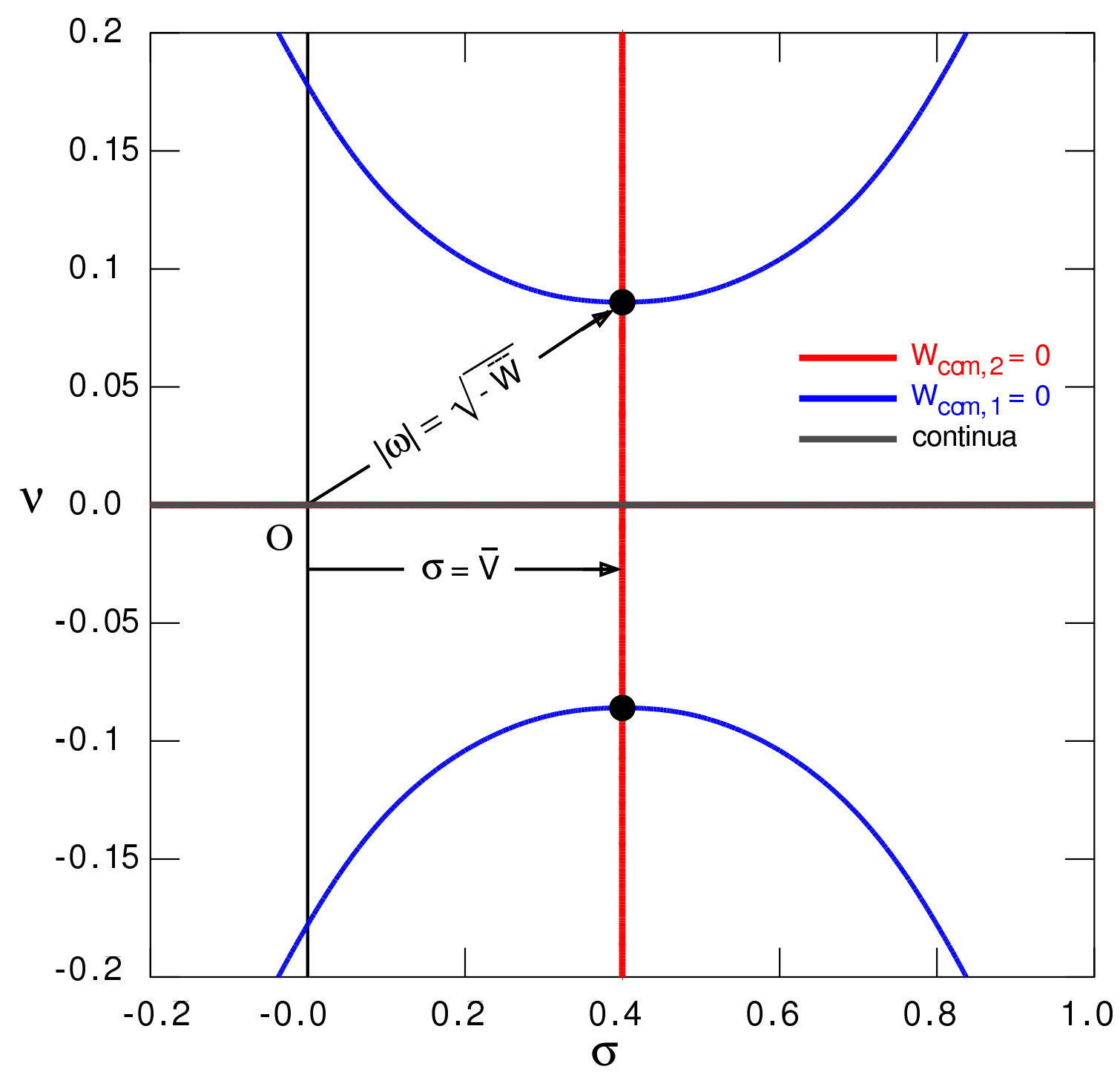

FIG. 1: Elementary Spectral Web consisting of solution path (red) and conjugate path (blue) with eigenvalues (black dots) at the intersections; forward and backward Alfvén continua are located on the real axis. [This example refers to the $m=1, k=1.28$ internal kink mode of a cylindrical force-free magnetic field of constant $\alpha a=8.0$, subjected to a constant Doppler shift, $\sigma=k v_{z}=0.4$.] 


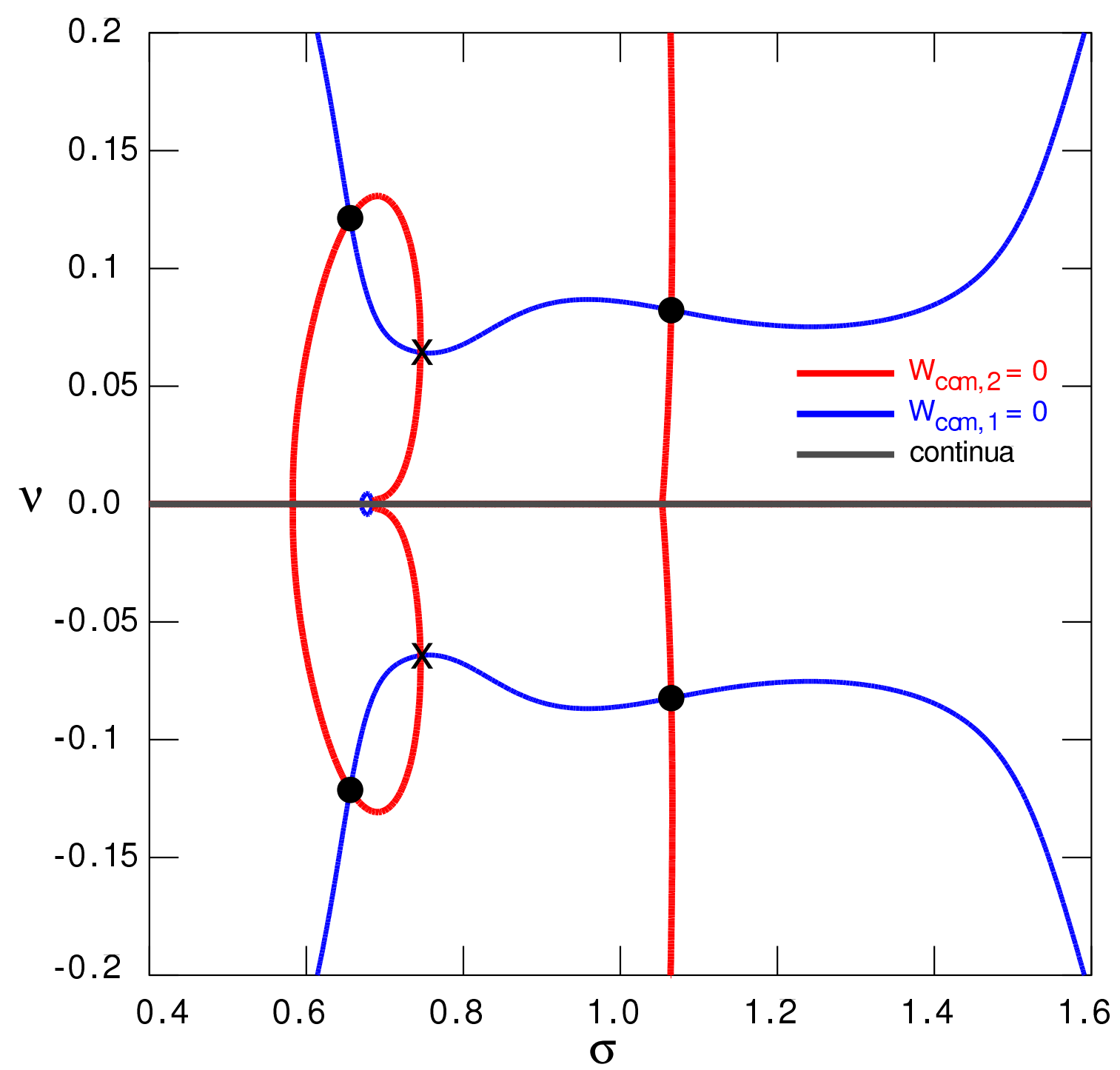

FIG. 2: Spectral Web of the same equilibrium as in Fig. 1, but subjected to a shear velocity profile $v_{z}=1-x^{2}$. Genuine eigenvalues are indicated by a dot, false ones by a cross. 